\title{
TOWARDS LAX FORMULATION OF INTEGRABLE HIERARCHIES OF TOPOLOGICAL TYPE
}

\author{
G. CARLET, J. VAN DE LEUR, H. POSTHUMA, AND S. SHADRIN
}

\begin{abstract}
To each partition function of cohomological field theory one can associate an Hamiltonian integrable hierarchy of topological type. The Givental group acts on such partition functions and consequently on the associated integrable hierarchies. We consider the Hirota and Lax formulations of the deformation of the hierarchy of $N$ copies of $\mathrm{KdV}$ obtained by an infinitesimal action of the Givental group. By first deforming the Hirota quadratic equations and then applying a fundamental lemma to express it in terms of pseudo-differential operators, we show that such deformed hierarchy admits an explicit Lax formulation. We then compare the deformed Hamiltonians obtained from the Lax equations with the analogous formulas obtained in [1,2], to find that they agree. We finally comment on the possibility of extending the Hirota and Lax formulation on the whole orbit of the Givental group action.
\end{abstract}

\section{Contents}

1. Introduction 2

1.1. CohFTs and hierarchies 2

1.2. Givental group action 2

1.3. Infinitesimal deformations of several copies of $\mathrm{KdV} \quad 3$

1.4. Organization of the paper 4

1.5. Acknowledgement 5

2. The KdV hierarchy 5

2.1. From Lax to Hirota 5

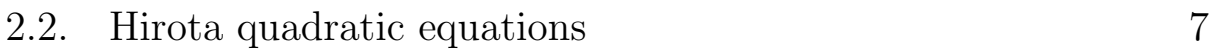

2.3. A fundamental lemma 8

2.4. From Hirota to Lax 9

2.5. The residues 10

2.6. Multiple copies of KdV 11

3. Givental-Lee infinitesimal deformations 13

3.1. Introduction to Givental's twisted loop group action 13

3.2. $R$-deformation of vertex operators 14

3.3. S-deformation of vertex operators $\quad 15$

3.4. Deformed Hirota equations 16

3.5. Global Givental group action on vertex operators 17

4. Deformations of the Sato-Wilson equations 18

4.1. Equations for the deformed wave functions 18 
4.2. Consequences of the Fundamental Lemma 19

4.3. The Lax operator 19

4.4. The deformed Sato-Wilson equations 20

4.5. An alternative form of the deformed Sato-Wilson equations 20

4.6. Explicit formulas for $Q_{\alpha, G}$ and $Q_{\alpha, G} P_{\alpha, G}^{-1} \quad 21$

4.7. Explicit computation of $S$-deformations 23

4.8. Explicit computation of $R$-deformations $\quad 25$

5. Comparison with deformations in Hamiltonian form 27

5.1. The $R$-deformations $\quad 27$

5.2. The $S$-deformations 34

References $\quad 35$

\section{INTRODUCTION}

1.1. CohFTs and hierarchies. A cohomological field theory (Co$\mathrm{hFT}$ ) is a system of factorizable forms on the moduli spaces of curves. It serves as an axiomatic setting that captures essential algebraic structures behind Gromov-Witten theory. Meanwhile, there are more examples of CohFTs, for example, coming from quantum singularity theory in the sense of Fan-Jarvis-Ruan [6]. In genus 0 the notion of a CohFT is equivalent to a solution of the WDVV equation and, under some extra assumptions, turns out to be equivalent to the notion of (a formal germ of) a Frobenius manifold.

To an arbitrary CohFT one can associate a hierarchy of PDEs in (infinite-dimensional) Hamiltonian form. It was first constructed by Dubrovin and Zhang in the semi-simple homogeneous case [4], and their construction gives, indeed, a bi-Hamiltonian hierarchy based solely on genus 0 data with some extra assumptions on dispersive behavoir of the tau function. In a weaker form, this construction is revisited in the recent papers of Buryak et al. [1, 2], where a Hamiltonian hierarchy was constructed using topological properties of the moduli spaces of curves in all genera.

In some special situations, related to singularity theory, there is an alternative construction of integrable hierarchies due to Givental and Milanov [12]. They define a hierarchy in terms of Hirota quadratic equations (HQEs), where the vertex operators are defined in terms of the period integrals of the Lefschetz thimbles of the corresponding singularity. Their constructions are always presented ad hoc, and we are interested to understand whether we can use their ideas in a wider range of examples. A separate question is whether we can identify, in some explicit way, their HQEs with the Hamiltonian equations of Dubrovin and Zhang.

1.2. Givental group action. The main tool used for the analysis of the integrable hierarchies in both approaches is the Givental theory of 
the group action on cohomological field theories. In the Hamiltonian formulation, we have a well-defined action on the Hamiltonians, the Poisson bracket, and the equations of the hierarchy. The infinitesimal deformations that correspond to the action of the Lie algebra of the Givental group are written down explicitly in $[1,2]$.

In the case of HQEs of Givental and Milanov [12] (and also [15, $16,17])$, the situation is a bit subtle. It is clear how to conjugate vertex operators with the elements of the Givental group, and this is exactly what they do in order to prove that a particular formal tau function satisfies the HQEs that they present. They specify an element of the Givental group that takes the tau function of several copies of $\mathrm{KdV}$ to a particular tau function that they consider. Then they conjugate the explicitly written vertex operators with this element of the Givental group, and show that absence of singularities near the potentially singular points is equivalent to the HQEs of (several copies of) the KdV hierarchy.

The problem is that this procedure doesn't work in the opposite direction. If we take the simplest vertex operators needed to present the KdV hierarchy in form of the HQEs and try to conjugate them with an element of the Givental group, we get some series whose coefficients are divergent infinite sums.

1.3. Infinitesimal deformations of several copies of $\mathrm{KdV}$. In this paper we perform a first step towards the understanding of the connection between the two approaches mentioned above: the action of the Givental group on HQEs of different hierarchies and the comparison of these hierarchies with Dubrovin-Zhang hierarchies known in Hamiltonian form. Namely, we look at the infinitesimal deformations of several copies of the KdV hierarchy.

The action of the Lie algebra of the Givental group on the HQEs of $\mathrm{KdV}$ is well-defined, that is, it doesn't lead to divergent infinite sums. We also have explicit formulas for the action of the same Lie algebra on the Hamiltonian form of $\mathrm{KdV}$. We translate these two actions from different sides to the Lax formulation of KdV and identify them modulo the ideal of $\mathrm{KdV}$ equations.

Of course, it is in some sense a very expected result, since we compare two hierarchies of PDEs (two deformations of several copies of KdV) that have a common solution, and, moreover, are constructed in terms of this common solution. However, the comparative analysis of these two deformations turns out to be quite non-trivial, and there is an actual difference since only one of these tangent actions integrates to a group action. 
We hope that our explicit computation can also give an idea of what kind of "renormalization" should be applied in order to have a welldefined action of the Givental group on the Hirota quadratic formulation of the hierarchies.

The fact that the Hirota quadratic formulation of the integrable hierarchies does not extend to arbitrary hierarchies associated to CohFTs is not very surprising from the point of view of the usual theory of integrable equations. On one hand the Dubrovin-Zhang construction [4] (and the weaker construction from CohFTs of [1,2]) produces families of integrable hierarchies with good properties (bi-Hamiltonian structure, existence of tau function) which depend on a large number of parameters (e.g. the charge $d$ parametrizes the conformal 2-dimensional Frobenius manifolds and therefore the corresponding Dubrovin-Zhang hierarchies with two dependent variables). On the other hand HQEs are only known for a much smaller subset of explicitly known hierarchies, typically those obtained as reductions of KP and 2D Toda type hierarchies. By "explicitly known" we mean hierarchies which can be represented in Lax form, which is usually given in terms of pseudodifferential or difference operators.

A natural question therefore arises, if it is possible to extend not only the Hirota, but also the Lax formulation to more general families of integrable hierarchies, e.g. to CohFT hierarchies on the orbit of the Givental group action containing $N$-copies of $\mathrm{KdV}$.

Our results, while only concerned with the infinitesimal deformations, demonstrate some progress in this direction and show several interesting features. First, we prove that it is indeed possible to express the deformed equations in Lax (or, equivalently Sato-Wilson) form using pseudo-differential operators. Second, while most examples of integrable hierarchies for which the Lax formulation is known are written in terms of a single Lax operator, our deformed equations feature $N$ Lax operators. Third, while we start by deforming second order differential operators of $\mathrm{KdV}$ type, the deformed operators are pseudodifferential operators where the non-trivial integral part is completely determined by the differential part. This suggests to view the deformed Lax equations as a sort of reduction of a deformation of multiple copies of the KP hierarchy.

We expect that this approach will be very helpful in the construction of the Lax pairs and HQEs formulations for at least some subclass of the Dubrovin-Zhang hierarchies.

1.4. Organization of the paper. In section 2 we recall some basic facts about the KdV hierarchy and its Lax and Hirota formulations. In particular we review in detail how to recover, using a fundamental lemma, the Sato-Wilson and Lax equations from the Hirota bilinear equations, first in the case of the single $\mathrm{KdV}$ hierarchy and than in 
the case of $n$-copies of KdV. In section 2 we compute the deformations of the vertex operators induced by the infinitesimal Givental action of the twisted loop group. We also comment on the possibility of computing the global Givental group action on the vertex operators. Explicit formulas for the deformed Sato-Wilson and Lax equations are obtained in section 4 by a careful application of the fundamental lemma to the deformed Hirota equations. Finally, in section 5, the deformation formulas for Hamiltonians, recovered as residues of the Lax operators, are shown to coincide with those obtained in [1].

1.5. Acknowledgement. G.C. wishes to acknowledge the support of the GQT cluster and of ESF - MISGAM grants. S.S. was supported by a Vidi grant of the Netherlands Organization for Scientific Research.

\section{The KDV HIERARCHY}

We briefly recall some basic constructions in the theory of the $\mathrm{KdV}$ hierarchy using the pseudo-differential operator formalism. First we sketch the definition of Lax, Sato-Wilson and Hirota quadratic equations, omitting most proofs. Then we give a more careful treatment of the derivation of Lax and Sato-Wilson equations from the Hirota equations, since this will be required later.

For more details see e.g. [3].

2.1. From Lax to Hirota. The KdV hierarchy is a sequence of commuting flows in Lax form

$$
\frac{\partial L}{\partial q_{n}}=\frac{a_{n}}{\sqrt{\hbar}}\left[\left(L^{n+\frac{1}{2}}\right)_{+}, L\right], \quad n=0,1,2, \ldots
$$

on the space of Lax operators

$$
L=\hbar \partial^{2}+2 u(x) \quad \text { with } \partial:=\frac{\partial}{\partial x} .
$$

Here $u(x)$ will be seen as a formal power series in $\sqrt{\hbar}$, i.e. $u(x ; \sqrt{\hbar})=$ $u_{0}(x)+u_{1}(x) \sqrt{\hbar}+\ldots$

Note that a solution $L=L(x, q)$ will be a function of $x$ and of all the times of the hierarchy $q=\left(q_{0}, q_{1}, \ldots\right)$.

The pseudo-differential operator $L^{\frac{1}{2}}$ is the square root of $L$ (i.e. the unique operator $L^{\frac{1}{2}}=\sqrt{\hbar} \partial+\ldots$ such that $\left.\left(L^{\frac{1}{2}}\right)^{2}=L\right)$ and is equal to

$$
\begin{aligned}
L^{\frac{1}{2}}= & \sqrt{\hbar} \partial+\frac{u}{\sqrt{\hbar}} \partial^{-1}-\frac{u^{\prime}}{2 \sqrt{\hbar}} \partial^{-2} \\
& +\frac{\hbar u^{\prime \prime}-2 u^{2}}{4 \hbar^{3 / 2}} \partial^{-3}-\frac{\hbar u^{(3)}-12 u u^{\prime}}{8 \hbar^{3 / 2}} \partial^{-4} \\
& +\frac{\hbar^{2} u^{(4)}-28 \hbar u u^{\prime \prime}-22 \hbar\left(u^{\prime}\right)^{2}+8 u^{3}}{16 \hbar^{5 / 2}} \partial^{-5}+\cdots .
\end{aligned}
$$


Given a pseudo-differential operator $A$, i.e. a Laurent series $A=$ $\sum_{n=-\infty}^{m} a_{n}(x) \partial^{n}$ for arbitrary $m$, we denote its differential part by $A_{+}:=\sum_{n=0}^{m} a_{n}(x) \partial^{n}$, while $A_{-}:=A-A_{+}$. The product of pseudodifferential operators is defined by

$$
\partial^{k} f(x)=\sum_{l=0}^{\infty}\left(\begin{array}{l}
k \\
l
\end{array}\right) \frac{\partial^{k} f}{\partial x^{k}} \partial^{k-l} .
$$

By $a_{n}, n=0,1,2, \ldots$, we denote the constants

$$
a_{n}=\frac{1}{(2 n+1) ! !} \text {. }
$$

The commutativity of the flows defined by the Lax equations (1) follows from the so-called Zakharov-Shabat (or zero curvature) equations

$$
\frac{\sqrt{\hbar}}{a_{k}} \frac{\partial\left(L^{\ell+\frac{1}{2}}\right)_{+}}{\partial q_{k}}-\frac{\sqrt{\hbar}}{a_{\ell}} \frac{\partial\left(L^{k+\frac{1}{2}}\right)_{+}}{\partial q_{\ell}}=\left[\left(L^{k+\frac{1}{2}}\right)_{+},\left(L^{\ell+\frac{1}{2}}\right)_{+}\right] .
$$

Note that in this equation we can change all projectors $(\cdot)_{+}$to $-(\cdot)_{-}$.

To a solution $L$ to the Lax equations (1) one can associate a dressing operator, i.e. a pseudo-differential operator of the form

$$
P(\sqrt{\hbar} \partial)=P(x, q, \sqrt{\hbar} \partial)=1+p_{1}(x, q)(\sqrt{\hbar} \partial)^{-1}+\ldots
$$

such that

$$
L=P(\sqrt{\hbar} \partial) \hbar \partial^{2} P(\sqrt{\hbar} \partial)^{-1}
$$

which satisfies the Sato-Wilson equations for the KdV hierarchy

$$
\frac{\partial P(\sqrt{\hbar} \partial)}{\partial q_{n}}=-\frac{a_{n}}{\sqrt{\hbar}}\left(P(\sqrt{\hbar} \partial)(\sqrt{\hbar} \partial)^{2 n+1} P(\sqrt{\hbar} \partial)^{-1}\right)_{-} P(\sqrt{\hbar} \partial) .
$$

On the other hand a solution (7) to Sato-Wilson equations (9) which satisfies the constraint

$$
\left(P(\sqrt{\hbar} \partial) \hbar \partial^{2} P(\sqrt{\hbar} \partial)^{-1}\right)_{-}=0
$$

defines, through (8), a solution $L$ to the KdV hierarchy.

Let us define the vertex operator $\Gamma$ as

$$
\Gamma(q, \lambda)=\Gamma_{+}(q, \lambda) \Gamma_{-}(q, \lambda)
$$

where

$$
\begin{aligned}
& \Gamma_{-}(q, \lambda)=\exp \left(-\sqrt{\hbar} \sum_{n=0}^{\infty} b_{n} \lambda^{-2 n-1} \frac{\partial}{\partial q_{n}}\right), \\
& \Gamma_{+}(q, \lambda)=\exp \left(\frac{1}{\sqrt{\hbar}} \sum_{n=0}^{\infty} a_{n} \lambda^{2 n+1} q_{n}\right) .
\end{aligned}
$$

Here, the constants $b_{n}$ are given by

$$
b_{n}:=(2 n-1) ! ! \quad n=0,1, \ldots
$$


The wave function is defined as

$$
\psi(x, q, \lambda)=P(x, q, \lambda) \Gamma_{+}(q, \lambda) e^{\frac{x \lambda}{\sqrt{\hbar}}} .
$$

Note that here we have denoted by $P(x, q, \lambda)$ the (right) symbol of the dressing operator (7)

$$
P(\lambda)=P(x, q, \lambda)=1+p_{1}(x, q) \lambda^{-1}+\ldots
$$

The wave function satisfies the following linear system

$$
\begin{aligned}
& L \psi(x, q, \lambda)=\lambda^{2} \psi(x, q, \lambda), \\
& \frac{\partial \psi(x, q, \lambda)}{\partial q_{n}}=\frac{a_{n}}{\sqrt{\hbar}}\left(L^{n+\frac{1}{2}}\right)_{+}(\psi(x, q, \lambda)) .
\end{aligned}
$$

It is well known that the wave function also satisfies the following system of quadratic equations

$$
\operatorname{Res}_{\lambda} \lambda^{2 p} \psi(x, q, \lambda) \psi\left(x^{\prime}, q^{\prime},-\lambda\right) d \lambda=0, \quad p=0,1,2, \ldots,
$$

where $\operatorname{Res}_{\lambda} \sum_{i} a_{i} \lambda^{i} d \lambda=a_{-1}$.

The KdV hierarchy can also be expressed in terms of the tau function. In particular one can prove that for any solution $P$ to the the Sato-Wilson equations (9) there exists a function $\tau=\tau(x, q)$ such that

$$
P(x, q, \lambda)=\frac{\Gamma_{-}(q, \lambda) \tau(x, q)}{\tau(x, q)} .
$$

Note that the tau function is uniquely determined by a solution of the Sato-Wilson equation up to a multiplicative constant.

The wave function (15) is given in terms of the tau function by

$$
\psi(x, q, \lambda)=\frac{\Gamma(q, \lambda) \tau(x, q)}{\tau(x, q)} e^{\frac{x \lambda}{\sqrt{\hbar}}} .
$$

It is obvious from the quadratic equations for the wave function (18) that the tau function also satisfies similar quadratic equations, viz., (21)

$$
\operatorname{Res}_{\lambda} \lambda^{2 p}(\Gamma(q, \lambda) \tau(x, q))\left(\Gamma\left(q^{\prime},-\lambda\right) \tau\left(x^{\prime}, q^{\prime}\right)\right) d \lambda=0, \quad p=0,1,2, \ldots
$$

These are called Hirota quadratic equations for the tau function. We proceed to consider them in more detail.

2.2. Hirota quadratic equations. The Hirota quadratic equations (HQEs) for the KdV hierarchy can be written in compact form as

$$
\operatorname{Res}_{\lambda} \lambda^{2 p}(\Gamma(\lambda) \otimes \Gamma(-\lambda))(\tau \otimes \tau) d \lambda=0 \quad p \geq 0 .
$$

The HQEs encode an infinite number of quadratic relations for the function $\tau$ and its derivatives w.r.t. the variables $q=\left(q_{0}, q_{1}, \ldots\right)$.

We will assume that the function $\tau(q)$ is a formal power series in the variables $q$ and, in particular, that it is of the form

$$
\tau(q)=e^{\hbar^{-1} \mathcal{F}(q ; \sqrt{\hbar})}
$$


for $\mathcal{F}(q ; \sqrt{\hbar})$ a formal power series in $q$ and $\sqrt{\hbar}$.

Equation (22) is interpreted as follows. After evaluating the two factors of the tensor product in $q$ and $q^{\prime}$, respectively, it is written as

$$
\operatorname{Res}_{\lambda} \lambda^{2 p}(\Gamma(q, \lambda) \tau(q))\left(\Gamma\left(q^{\prime},-\lambda\right) \tau\left(q^{\prime}\right)\right) d \lambda=0, \quad p=0,1,2, \ldots
$$

The vertex operator $\Gamma(q, \lambda)$ has been defined in (11)-(13). Passing to the variables $\eta, \xi$ defined by

$$
q_{n}=\xi_{n}+\eta_{n}, \quad q_{n}^{\prime}=\xi_{n}-\eta_{n}
$$

one rewrites the argument of the residue as $\lambda^{2 p}$ multiplied by

$$
e^{\frac{2}{\sqrt{\hbar}} \sum_{n \geq 0} a_{n} \lambda^{2 n+1} \eta_{n}} e^{-\sqrt{\hbar} \sum_{n \geq 0} b_{n} \lambda^{-2 n-1} \frac{\partial}{\partial \eta_{n}}} \tau(\xi+\eta) \tau(\xi-\eta) .
$$

This is a formal power series in the variables $\eta$ with coefficients which are Laurent series in $\lambda^{-1}$, i.e., with upper bounded powers of $\lambda$. The HQEs (22) state that in each of these Laurent series the terms with odd negative powers of $\lambda$ are vanishing.

We can also express the Hirota equations in terms of regularity of the differential 1 -form

$$
(\Gamma(\lambda) \otimes \Gamma(-\lambda)-\Gamma(-\lambda) \otimes \Gamma(\lambda))(\tau \otimes \tau) d \lambda .
$$

We say that this 1 -form is regular in $\lambda$ if, after the change of variables and the expansion in $\eta$ as before, the resulting Laurent series have no polar part, i.e. they are polynomials in $\lambda$. Since the averaging in (27) kills exactly the even terms, this is equivalent to the vanishing of the odd negative powers of $\lambda$ in (26). Hence $\tau(q)$ satisfies the Hirota quadratic equations for $\mathrm{KdV}$ iff the 1 -form (27) is regular.

2.3. A fundamental lemma. To derive the Sato-Wilson equations from the HQEs we need the following Fundamental Lemma which will also be important in Section 3 .

Lemma 2.1. (Fundamental Lemma) Let $P(x, \sqrt{\hbar} \partial)$ and $Q(x, \sqrt{\hbar} \partial)$ be two pseudo-differential operators. Then the equation

$$
\operatorname{Res}_{\lambda} P(x, \lambda) e^{\frac{x \lambda}{\sqrt{\hbar}}} Q\left(x^{\prime},-\lambda\right) e^{-\frac{x^{\prime} \lambda}{\sqrt{\hbar}}} d \lambda=0
$$

is equivalent to

$$
\left(P(x, \sqrt{\hbar} \partial) \cdot Q(x, \sqrt{\hbar} \partial)^{*}\right)_{-}=0 .
$$

Here $P(x, \lambda)$ denotes the right symbol of $P(x, \sqrt{\hbar} \partial)$ and $Q^{*}$ is the formal adjoint of $Q$, defined by $\left(c(x) \partial^{k}\right)^{*}=(-\partial)^{k} \cdot c(x)$.

We give a short proof of this Lemma, along long the lines of [3] and [7].

Proof. The proof is based on the following identity, which can be checked by direct computation

$$
\operatorname{Res}_{\lambda} P(x, \lambda) Q(x,-\lambda) d \lambda=\sqrt{\hbar} \operatorname{Res}_{\partial} P(x, \sqrt{\hbar} \partial) \cdot Q(x, \sqrt{\hbar} \partial)^{*} .
$$


Equation (29) is equivalent to

$$
\operatorname{Res}_{\partial} P(x, \sqrt{\hbar} \partial) \cdot Q(x, \sqrt{\hbar} \partial)^{*} \cdot \partial^{k}=0 \quad \text { for each } k \geq 0
$$

or, introducing an extra formal variable $y$, to the generating identity

$$
\operatorname{Res}_{\partial} P(x, \sqrt{\hbar} \partial) \cdot Q(x, \sqrt{\hbar} \partial)^{*} \cdot e^{-y \partial}=0 .
$$

Noting that

$$
\begin{aligned}
Q(x, \sqrt{\hbar} \partial)^{*} \cdot e^{-y \partial} & =\left(e^{y \partial} \cdot Q(x, \sqrt{\hbar} \partial)\right)^{*} \\
& =\left(Q(x+y, \sqrt{\hbar} \partial) \cdot e^{y \partial}\right)^{*}
\end{aligned}
$$

and making use of equation (30), we can rewrite equation (32) as

$$
\operatorname{Res}_{\lambda} P(x, \lambda) Q(x+y,-\lambda) e^{-\frac{y \lambda}{\sqrt{\hbar}}} d \lambda=0 .
$$

After a change of variable $y=x^{\prime}-x$, this is exactly equation (28).

2.4. From Hirota to Lax. Let $\tau(q)$ be a solution to the Hirota quadratic equations (24). Let us introduce a dependence on $x$ (and $x^{\prime}$ ) by shifting $q_{0} \rightarrow q_{0}+x$ and $q_{0}^{\prime} \rightarrow q_{0}^{\prime}+x^{\prime}$. Denote $\tau(x, q)=\tau\left(q_{0}+x, q_{1}, \ldots\right)$ and

$$
P(x, q, \lambda)=\frac{\Gamma_{-}(q, \lambda) \tau(x, q)}{\tau(x, q)} .
$$

Substituting in (24) and dividing by $\tau(x, q) \tau\left(x^{\prime}, q^{\prime}\right)$ we obtain

$$
\operatorname{Res}_{\lambda}\left(\lambda^{2 p} \Gamma_{+}(q, \lambda) P(x, q, \lambda) e^{\frac{x}{\sqrt{\hbar}} \lambda} \Gamma_{+}\left(q^{\prime},-\lambda\right) P\left(x^{\prime}, q^{\prime},-\lambda\right) e^{-\frac{x^{\prime}}{\sqrt{\hbar}} \lambda}\right) d \lambda=0 .
$$

Using the Fundamental Lemma we rewrite the HQEs as the following bilinear equation involving pseudo-differential operators

$$
\left(P(x, q, \sqrt{\hbar} \partial) \Gamma_{+}(q, \sqrt{\hbar} \partial) \cdot \hbar^{p} \partial^{2 p} \cdot \Gamma_{+}\left(q^{\prime}, \sqrt{\hbar} \partial\right)^{*} P\left(x, q^{\prime}, \sqrt{\hbar} \partial\right)^{*}\right)_{-}=0 .
$$

Let us examine some consequences of this equation.

First, set $p=0$ and $q=q^{\prime}$. Since $\Gamma_{+}(q, \sqrt{\hbar} \partial)^{*}=\Gamma_{+}(q, \sqrt{\hbar} \partial)^{-1}$, one finds

$$
\left(P(x, q, \sqrt{\hbar} \partial) P(x, q, \sqrt{\hbar} \partial)^{*}\right)_{-}=0 .
$$

This implies, together with the fact that, by the definition $(36), P(x, q, \sqrt{\hbar} \partial)=$ $1+(P(x, q, \sqrt{\hbar} \partial))_{-}$, that

$$
P(x, q, \sqrt{\hbar} \partial)^{*}=P(x, q, \sqrt{\hbar} \partial)^{-1} .
$$

Second, for $p=1$ and $q=q^{\prime}$ we have

$$
\left(P(x, q, \sqrt{\hbar} \partial) \hbar \partial^{2} P(x, q, \sqrt{\hbar} \partial)^{-1}\right)_{-}=0 .
$$


This implies that the pseudo-differential operator defined by

$$
L=L(x, q, \sqrt{\hbar} \partial):=P(x, q, \sqrt{\hbar} \partial) \hbar \partial^{2} P(x, q, \sqrt{\hbar} \partial)^{-1},
$$

is actually second order differential operator of the form

$$
L=\hbar \partial^{2}+2 u(x, q) \text {. }
$$

This gives immediately that

$$
u=\hbar \frac{\partial^{2} \log \tau}{\partial x \partial q_{0}} .
$$

Third, by differentiating equation (38) w.r.t. $q_{n}$ and setting $q=q^{\prime}$ (and $p=0$ ), since

$$
\frac{\partial \Gamma_{+}(q, \sqrt{\hbar} \partial)}{\partial q_{n}}=\frac{a_{n}}{\sqrt{\hbar}}(\sqrt{\hbar} \partial)^{2 n+1} \Gamma_{+}(q, \sqrt{\hbar} \partial),
$$

we obtain the Sato-Wilson equations

$$
\frac{\partial P(\sqrt{\hbar} \partial)}{\partial q_{n}} P(\sqrt{\hbar} \partial)^{-1}=-\frac{a_{n}}{\sqrt{\hbar}}\left(P(\sqrt{\hbar} \partial)(\sqrt{\hbar} \partial)^{2 n+1} P(\sqrt{\hbar} \partial)^{-1}\right)_{-}
$$

Here and in the following we omit explicit dependence on $x, q$ when clear from the context.

As explained before, the Lax equations (1) are an immediate consequence of the Sato-Wilson equations. Summarizing, we have proved that a tau function $\tau(q)$ which satisfies the HQEs defines a solution $u(x, q)$ of the KdV hierarchy.

2.5. The residues. Here we collect some observations on the residues that will be needed later. Taking the residue of (36) we find

$$
\operatorname{Res}_{\partial} P(\sqrt{\hbar} \partial)=-\frac{\partial \log \tau}{\partial q_{0}}
$$

If we use this together with the residue of the Sato-Wilson equation (46), we obtain

$$
\frac{a_{n}}{\sqrt{\hbar}} \operatorname{Res}_{\partial} L^{n+\frac{1}{2}}=\frac{\partial^{2} \log \tau}{\partial q_{0} \partial q_{n}}
$$

Differentiating this equation w.r.t. $q_{m}$ we obtain

$$
\frac{a_{m} a_{n}}{\hbar} \operatorname{Res}_{\partial}\left[\left(L^{m+\frac{1}{2}}\right)_{+},\left(L^{n+\frac{1}{2}}\right)_{-}\right]=\frac{\partial^{3} \log \tau}{\partial q_{0} \partial q_{m} \partial q_{n}} .
$$

Note that, since the dependence of $\tau$ on $x, q_{0}$ is only through $q_{0}+x$, we can always replace the derivative w.r.t. $q_{0}$ with the derivative w.r.t. $x$ and vice-versa. 
2.6. Multiple copies of $\mathbf{K d V}$. By definition, the tau function of $N$ copies of the KdV hierarchy is the product

$$
\tau(q)=\prod_{\gamma=1}^{N} \tau_{\gamma}\left(q_{\gamma}\right)
$$

where each $\tau_{\gamma}\left(q_{\gamma}\right)$ is a tau function of the $\mathrm{KdV}$ hierarchy, depending only on the variables $q_{\gamma}=\left(q_{\gamma, 0}, q_{\gamma, 1}, q_{\gamma, 2}, \ldots\right)$. We denote by $q$ the totality of the variables $q_{\gamma, n}$ for $\gamma=1, \ldots, N$ and $n \geq 0$.

Since each of the factors $\tau_{\gamma}\left(q_{\gamma}\right)$ satisfies the HQEs of KdV (22) with respect to the variables $q_{\gamma}$, the tau function $\tau(q)$ satisfies the same $N$ HQEs.

Let us express this system of HQEs as a single equation. Let the variables $\lambda_{\alpha}$ be the local coordinates $\lambda-z_{\alpha}$ near some points $z_{\alpha} \in \mathbb{C}$, $\alpha=1, \ldots, N$. The system of Hirota quadratic equations can be written as

$$
\operatorname{Res}_{\lambda_{\alpha}} \lambda_{\alpha}^{2 p} \Gamma_{\alpha}\left(\lambda_{\alpha}\right) \tau(q) \otimes \Gamma_{\alpha}\left(-\lambda_{\alpha}\right) \tau(q) d \lambda=0,
$$

where $p \geq 0$ and $\alpha=1, \ldots, N$. Recall that the tensor product means that we are evaluating the two factors in two different sets of variables $q$ and $q^{\prime}$. Here $\Gamma_{\alpha}\left(\lambda_{\alpha}\right)=\Gamma_{\alpha,+}\left(\lambda_{\alpha}\right) \Gamma_{\alpha,-}\left(\lambda_{\alpha}\right)$, and

$$
\begin{aligned}
& \Gamma_{\alpha,-}\left(\lambda_{\alpha}\right)=\exp \left(-\sqrt{\hbar} \sum_{n=0}^{\infty} b_{n} \lambda_{\alpha}^{-2 n-1} \frac{\partial}{\partial q_{\alpha, n}}\right), \\
& \Gamma_{\alpha,+}\left(\lambda_{\alpha}\right)=\exp \left(\frac{1}{\sqrt{\hbar}} \sum_{n=0}^{\infty} a_{n} \lambda_{\alpha}^{2 n+1} q_{\alpha, n}\right),
\end{aligned}
$$

are the vertex operators of the KdV hierarchy, acting of the variables $q_{\alpha}$ and evaluated in the variables $\lambda_{\alpha}=\lambda-z_{\alpha}$.

As before we can reformulate the Hirota equations for $N$ copies of $\mathrm{KdV}$ as regularity of the differential 1 -form in $\lambda$

$$
\sum_{\alpha=1}^{N}\left(\Gamma_{\alpha}\left(\lambda_{\alpha}\right) \otimes \Gamma_{\alpha}\left(-\lambda_{\alpha}\right)-\Gamma_{\alpha}\left(-\lambda_{\alpha}\right) \otimes \Gamma_{\alpha}\left(\lambda_{\alpha}\right)\right)(\tau \otimes \tau) d \lambda
$$

where $\lambda_{\alpha}=\lambda-z_{\alpha}$. Regularity in this case means that the Laurent series expansions around each point $z_{\alpha} \in \mathbb{C}$ have no polar part. In other words, $\tau(q)$ satisfies the Hirota quadratic equations for $N$ copies of the KdV hierarchy iff (53) is holomorphic as a function of $\lambda \in \mathbb{C}$.

Let's now obtain the Sato-Wilson and Lax equations from Hirota equations for $N$ copies of the $\mathrm{KdV}$ hierarchy. Let $\tau(q)$ be a solution of HQEs (51) of the form (23).

As in the case of a single copy of the KdV hierarchy we have first to introduce a dependence of the space variable $x$ in the Hirota equations by shifting $q_{\alpha, 0} \rightarrow q_{\alpha, 0}+x$ for each $\alpha=1, \ldots, N$. 
Let

$$
P_{\alpha}(x, q, \lambda)=\frac{\Gamma_{\alpha,-}(\lambda) \tau(x, q)}{\tau(x, q)}
$$

for $\alpha=1, \ldots, N$.

Substituting in the Hirota equations and applying the fundamental lemma we obtain the following bilinear equations in terms of pseudodifferential operators

$\left(P_{\alpha}(x, q, \sqrt{\hbar} \partial) \Gamma_{\alpha,+}(q, \sqrt{\hbar} \partial) \cdot \hbar^{p} \partial^{2 p} \cdot \Gamma_{\alpha,+}\left(q^{\prime}, \sqrt{\hbar} \partial\right)^{*} P_{\alpha}\left(x, q^{\prime}, \sqrt{\hbar} \partial\right)^{*}\right)_{-}=0$

for $\alpha=1, \ldots, N$.

Setting $p=1$ and $q=q^{\prime}$ as before, we get the constraints

$$
\left(P_{\alpha}(x, q, \sqrt{\hbar} \partial) \hbar \partial^{2} P_{\alpha}(x, q, \sqrt{\hbar} \partial)^{-1}\right)_{-}=0 .
$$

It follows that the hierarchy is described by $N$ Lax operators given by

$$
L_{\alpha}=P_{\alpha}(x, q, \sqrt{\hbar} \partial) \hbar \partial^{2} P_{\alpha}(x, q, \sqrt{\hbar} \partial)^{-1}=\hbar \partial^{2}+2 u_{\alpha}(x, q) .
$$

The Sato-Wilson equations are obtained by differentiating (55) w.r.t. $q_{\beta, n}$ and setting $q=q^{\prime}$. Since $\Gamma_{\alpha,+}(q, \sqrt{\hbar} \partial)$ depends only on the variables $q_{\alpha}$, we see that these equations decouple, as expected:

$\frac{\partial P_{\alpha}(\sqrt{\hbar} \partial)}{\partial q_{\beta, n}} P_{\alpha}(\sqrt{\hbar} \partial)^{-1}=-\delta_{\alpha, \beta} \frac{a_{n}}{\sqrt{\hbar}}\left(P_{\alpha}(\sqrt{\hbar} \partial)(\sqrt{\hbar} \partial)^{2 n+1} P_{\alpha}(\sqrt{\hbar} \partial)^{-1}\right)_{-}$.

Hence the dressing operator $P_{\alpha}$ depends only on the variables $q_{\alpha}$, and this in turn implies that the tau function factorizes as a product (50) of tau functions of the KdV hierarchy.

The Lax equations obviously also decouple

$$
\frac{\partial L_{\alpha}}{\partial q_{\beta, n}}=\delta_{\alpha, \beta} \frac{a_{n}}{\sqrt{\hbar}}\left[\left(L_{\alpha}^{2 n+1}\right)_{+}, L_{\alpha}\right] .
$$

From (54) we get as before the following residue formulas

$$
\operatorname{Res}_{\partial} P_{\alpha}(\sqrt{\hbar} \partial)=-\frac{\partial \log \tau}{\partial q_{\alpha, 0}}
$$

and, using Sato-Wilson equations,

$$
\frac{\partial^{2} \log \tau}{\partial q_{\alpha, 0} q_{\beta, p}}=\delta_{\alpha \beta} \frac{a_{n}}{\sqrt{\hbar}} \operatorname{Res}_{\partial} L_{\alpha}^{n+\frac{1}{2}}
$$

Note that the factorization (50) of the tau function of multiple copies of $\mathrm{KdV}$ implies that the functions defined by

$$
\Omega_{\alpha, p ; \beta, q}(x, q)=\hbar \frac{\partial^{2} \log \tau}{\partial q_{\alpha, p} \partial q_{\beta, q}}
$$

are non-zero only for $\alpha=\beta$. 


\section{Givental-Lee infinitesimal Deformations}

In this section, after recalling the basic constructions of the Givental group action on tau functions, we compute the explicit expressions of the deformations of the vertex operators and of the Hirota quadratic equations.

3.1. Introduction to Givental's twisted loop group action. Let $V$ be an $N$ dimensional vector space equipped with a scalar product $\langle$,$\rangle . Fix an orthonormal basis \left\{e_{\alpha}\right\}, \alpha=1, \ldots, N$ and denote $\mathbb{1}=$ $\sum_{\alpha=1}^{N} e_{\alpha}$.

The symplectic form

$$
\Omega(f, g)=\operatorname{Res}_{z}\langle f(-z), g(z)\rangle d z
$$

defined on the space $\mathcal{V}=V\left(\left(z^{-1}\right)\right)$ of formal Laurent series in $z^{-1}$ with values in $V$, allows to identify $\mathcal{V}$ with the cotangent bundle $T^{*} \mathcal{V}_{+}$, thanks to the polarization $\mathcal{V}=\mathcal{V}_{+} \oplus \mathcal{V}_{-}$, where $\mathcal{V}_{+}=V[z]$ and $\mathcal{V}_{-}=$ $z^{-1} V\left[\left[z^{-1}\right]\right]$ are Lagrangian subspaces of $\mathcal{V}$. The functions $q_{\alpha, k}=$ $\Omega\left(e_{\alpha}(-z)^{-k-1}, \cdot\right)$ and $p_{\alpha, k}=\Omega\left(\cdot, e_{\alpha} z^{k}\right)$, for $\alpha=1, \ldots, N, k \geq 0$, define Darboux coordinates on $\mathcal{V}$.

The loop group $\mathcal{L} \mathrm{GL}(V)$, given by $\mathrm{GL}(V)$-valued formal functions of $z$, acts on $\mathcal{V}$. The elements of $\mathcal{L} \mathrm{GL}(V)$ which preserve the symplectic form $\Omega$ define the twisted loop group $\mathcal{L}^{(2)} \mathrm{GL}(V)$. The associated Lie algebra $\mathcal{L}^{(2)} \operatorname{End}(V)$ - given by the infinitesimal symplectic transformations of $\mathcal{V}$ - splits into two subalgebras

$$
\mathfrak{g}_{ \pm}=\left\{\mathfrak{u}(z)=\sum_{k>0} \mathfrak{u}_{k} z^{ \pm k}, \mathfrak{u}_{i} \in \operatorname{End}(V), \mathfrak{u}(-z)^{t}+\mathfrak{u}(z)=0\right\}
$$

respectively called upper-triangular $\mathfrak{g}_{+}$and lower-triangular $\mathfrak{g}_{-}$.

The symplectic transformations $G(z)=e^{\mathfrak{u}(z)} \in \mathcal{L}^{(2)} \mathrm{GL}(V)$ obtained by exponentiating elements $\mathfrak{u}(z) \in \mathfrak{g}_{ \pm}$define the so-called upper-triangular, $G_{+}$, and lower-triangular, $G_{-}$, subgroups of the twisted loop group. In the following we will typically denote by $R$ (resp. $S$ ) the elements of $G_{+}\left(\right.$resp. $\left.G_{-}\right)$.

We can associate to the elements of the twisted loop group some linear operators in the variables $q_{\alpha, p}$ by a quantization procedure which is performed in two steps.

First, the infinitesimal symplectic trasformation of $\mathcal{V}$ associated with $\mathfrak{u} \in \mathfrak{g}_{ \pm}$is a linear Hamiltonian vector field induced by the Hamiltonian $H_{\mathfrak{u}}(f)=\frac{1}{2} \Omega(f, \mathfrak{u} f), f \in \mathcal{V}$. The Hamiltonian $H_{\mathfrak{u}}$ can thus be written as a quadratic function in the Darboux variables $q_{\alpha, k}, p_{\alpha, k}$.

Second, $H_{\mathfrak{u}}$ can be quantized to give an operator $\hat{\mathfrak{u}}$ by the rule

$$
q_{\alpha, k} \mapsto \frac{1}{\sqrt{\hbar}} q_{\alpha, k}, \quad p_{\alpha, k} \mapsto \sqrt{\hbar} \frac{\partial}{\partial q_{\alpha, k}},
$$


with the usual ordering ambiguity fixed by

$$
q_{\alpha, p} p_{\beta, q} \mapsto q_{\alpha, p} \frac{\partial}{\partial q_{\beta, q}} .
$$

The quantization of an element $G=e^{\mathfrak{u}} \in G_{ \pm}$is defined by $\hat{G}=e^{\hat{\mathfrak{u}}}$.

Further information on Givental group action can be found in $[8,13$, 14].

3.2. $R$-deformation of vertex operators. Consider an element $\mathfrak{r}=$ $\mathfrak{r}_{\ell} z^{\ell}$ of the Lie algebra $\mathfrak{g}_{+}$of the twisted loop group, for fixed $\ell>0$. According to the quantization procedure described above, the corresponding differential operator is given, up to multiplication by $(-1)^{\ell-1}$, by

$$
\hat{\mathfrak{r}}=\sum_{\alpha, \beta=1}^{N}\left(\mathfrak{r}_{\ell}\right)_{\alpha \beta}\left(\sum_{i=0}^{\infty} q_{\alpha, i} \frac{\partial}{\partial q_{\beta, \ell+i}}+\frac{\hbar}{2} \sum_{i+j=\ell-1}(-1)^{i+1} \frac{\partial^{2}}{\partial q_{\alpha, i} \partial q_{\beta, j}}\right)
$$

where $\left(\mathfrak{r}_{\ell}\right)_{\alpha \beta}=(-1)^{\ell+1}\left(\mathfrak{r}_{\ell}\right)_{\beta \alpha}=\left\langle e_{\alpha}, \mathfrak{r}_{\ell}\left(e_{\beta}\right)\right\rangle$ is the matrix associated to $\mathfrak{r}_{\ell} \in \operatorname{End}(V)$. Let $\hat{R}=\exp (\epsilon \hat{\mathfrak{r}})$.

The action of the group element $\hat{R}$ on the vertex operators (and therefore the tangent action by the Lie algebra element $\hat{\mathfrak{r}}$ ) is given by

$$
\hat{R} \Gamma_{\alpha} \hat{R}^{-1}=\hat{R} \Gamma_{\alpha,+} \hat{R}^{-1} \hat{R} \Gamma_{\alpha,-} \hat{R}^{-1} .
$$

A direct computation gives, up to an order $\epsilon^{2}$ correction,

$$
\begin{aligned}
\hat{R} \Gamma_{\alpha,-}(\lambda) \hat{R}^{-1} & =\Gamma_{\alpha,-}(\lambda) \exp \left(\epsilon \sqrt{\hbar} \sum_{\beta, n \geq 0}\left(\mathfrak{r}_{\ell}\right)_{\alpha \beta} b_{n} \lambda^{-2 n-1} \frac{\partial}{\partial q_{\beta, n+\ell}}\right), \\
\hat{R} \Gamma_{\alpha,+}(\lambda) \hat{R}^{-1} & =\exp \left(\epsilon\left(\mathfrak{r}_{\ell}\right)_{\alpha \alpha} d_{\ell} \lambda^{2 \ell}\right) \Gamma_{\alpha,+}(\lambda) \\
& \times \exp \left(\frac{\epsilon(-1)^{\ell-1}}{\sqrt{\hbar}} \sum_{\beta}\left(\mathfrak{r}_{\ell}\right)_{\alpha \beta}\left(\sum_{n \geq \ell} a_{n} q_{\beta, n-\ell} \lambda^{2 n+1}\right)\right) \\
& \times \exp \left(\epsilon \sqrt{\hbar} \sum_{\beta}\left(\mathfrak{r}_{\ell}\right)_{\alpha \beta} \sum_{n=0}^{\ell-1}(-1)^{n+1} a_{n} \frac{\partial}{\partial q_{\beta, \ell-1-n}} \lambda^{2 n+1}\right),
\end{aligned}
$$

where

$$
d_{\ell}=\frac{1}{2} \sum_{i+j=\ell-1}(-1)^{j+1} a_{i} a_{j}= \begin{cases}0 & \text { if } \ell \text { even } \\ -\frac{a_{\ell-1}}{2 \ell} & \text { if } \ell \text { odd }\end{cases}
$$

To prove these identities observe that the vertex operators $\Gamma_{\alpha, \pm}(\lambda)$ are of the form $\exp \hat{\mathfrak{f}}_{ \pm}$for certain differential operators $\hat{\mathfrak{f}}_{ \pm}$, which can 
be read off from (52). Up to $O\left(\epsilon^{2}\right)$ one has

$$
\begin{aligned}
\hat{R} \Gamma_{\alpha, \pm}(\lambda) \hat{R}^{-1} & =e^{\epsilon \hat{\mathfrak{\epsilon}}} e^{\hat{\mathfrak{f}}_{ \pm}} e^{-\epsilon \hat{\mathfrak{r}}}=e^{\hat{\mathfrak{f}}_{ \pm}}\left(1+\epsilon e^{-\hat{\mathfrak{f}}_{ \pm}} \hat{\mathfrak{r}} e^{\hat{\mathfrak{f}}_{ \pm}}\right) e^{-\epsilon \hat{\mathfrak{r}}} \\
& =e^{\hat{\mathfrak{f}}_{ \pm}}\left(1+\epsilon \hat{\mathfrak{r}}+\epsilon \hat{\mathfrak{a}}_{ \pm}\right) e^{-\epsilon \hat{\mathfrak{r}}}=e^{\hat{\mathfrak{f}}^{\epsilon \hat{\mathfrak{a}}_{ \pm}}}=\Gamma_{\alpha, \pm}(\lambda) e^{\epsilon \hat{\mathfrak{a}}_{ \pm}}
\end{aligned}
$$

where the operators $\hat{\mathfrak{a}}_{ \pm}$are defined by

$$
e^{-\hat{\mathfrak{f}}_{ \pm}} \hat{\mathfrak{r}} e^{\hat{\mathfrak{f}}_{ \pm}}=\hat{\mathfrak{r}}+\hat{\mathfrak{a}}_{ \pm} .
$$

Convention 3.1. From now on we will make all calculations up to an order $\epsilon^{2}$ correction. The additive term $O\left(\epsilon^{2}\right)$ will always be implicitly assumed.

3.3. $S$-deformation of vertex operators. Consider an element $\mathfrak{s}=$ $\mathfrak{s}_{\ell} z^{-\ell}$ of the Lie algebra $\mathfrak{g}_{-}$, for fixed $\ell>0$, and its quantization

$$
\hat{\mathfrak{s}}=\sum_{\alpha, \beta=1}^{N}\left(\mathfrak{s}_{\ell}\right)_{\alpha \beta}\left((-1)^{\ell-1} \sum_{i=0}^{\infty} q_{\alpha, i+\ell} \frac{\partial}{\partial q_{\beta, i}}+\frac{1}{2 \hbar} \sum_{i+j=\ell-1}(-1)^{i} q_{\alpha, i} q_{\beta, j}\right) .
$$

Let $\hat{S}=\exp (\epsilon \hat{\mathfrak{s}})$.

Again, we define the action of the group element $\hat{S}$ (and therefore the tangent action by the Lie algebra element $\hat{\mathfrak{s}}$ ) as

$$
\hat{S} \Gamma_{\alpha} \hat{S}^{-1}=\hat{S} \Gamma_{\alpha,+} \hat{S}^{-1} \hat{S} \Gamma_{\alpha,-} \hat{S}^{-1}
$$

A direct computation gives

$$
\begin{aligned}
\hat{S} \Gamma_{\alpha,-}( & \lambda) \hat{S}^{-1}=\exp \left(\epsilon\left(\mathfrak{s}_{\ell}\right)_{\alpha \alpha} f_{\ell} \lambda^{-2 \ell}\right) \\
& \times \exp \left(\frac{\epsilon}{\sqrt{\hbar}} \sum_{\beta}\left(\mathfrak{s}_{\ell}\right)_{\alpha \beta} \sum_{n=0}^{\ell-1}(-1)^{n} b_{n} \lambda^{-2 n-1} q_{\beta, \ell-1-n}\right) \Gamma_{\alpha,-}(\lambda) \\
& \times \exp \left(\epsilon \sqrt{\hbar} \sum_{\beta}\left(\mathfrak{s}_{\ell}\right)_{\alpha \beta} \sum_{n \geq \ell}(-1)^{\ell-1} b_{n} \lambda^{-2 n-1} \frac{\partial}{\partial q_{\beta, n-\ell}}\right) \\
\hat{S} \Gamma_{\alpha,+}(\lambda) \hat{S}^{-1}=\Gamma_{\alpha,+}(\lambda) & \quad \times \exp \left(\frac{\epsilon}{\sqrt{\hbar}} \sum_{\beta, n \geq 0}\left(\mathfrak{s}_{\ell}\right)_{\alpha \beta} a_{n} \lambda^{2 n+1} q_{\beta, n+\ell}\right)
\end{aligned}
$$

where

$$
f_{\ell}=\frac{1}{2} \sum_{i+j=\ell-1}(-1)^{i+1} b_{i} b_{j}= \begin{cases}0 & \text { if } \ell \text { even } \\ -\frac{b_{\ell}}{2 \ell} & \text { if } \ell \text { odd }\end{cases}
$$


3.4. Deformed Hirota equations. To deform the Hirota equations (51) we act on it with the operator $\hat{R} \otimes \hat{R}$, obtaining

$$
\begin{aligned}
\operatorname{Res}_{\lambda_{\alpha}} \lambda_{\alpha}^{2 p}\left(\hat{R} \Gamma_{\alpha,+}\left(\lambda_{\alpha}\right) \hat{R}^{-1}\right)\left(\hat{R} \Gamma_{\alpha,-}\left(\lambda_{\alpha}\right) \hat{R}^{-1}\right) \hat{R} \tau(q) \\
\quad \otimes\left(\hat{R} \Gamma_{\alpha,+}\left(-\lambda_{\alpha}\right) \hat{R}^{-1}\right)\left(\hat{R} \Gamma_{\alpha,-}\left(-\lambda_{\alpha}\right) \hat{R}^{-1}\right) \hat{R} \tau(q) d \lambda_{\alpha}=0 .
\end{aligned}
$$

We then use the formulas for the $R$-deformations of the vertex operators computed in the previous section.

We define $R_{\alpha,+}(\lambda)$ and $R_{\alpha,-}(\lambda)$ by

$$
\begin{aligned}
R_{\alpha,+}(\lambda):= & \exp \left(\epsilon\left(\mathfrak{r}_{\ell}\right)_{\alpha \alpha} d_{\ell} \lambda^{2 \ell}\right) \\
& \times \exp \left(\frac{\epsilon(-1)^{\ell-1}}{\sqrt{\hbar}} \sum_{\beta}\left(\mathfrak{r}_{\ell}\right)_{\alpha \beta}\left(\sum_{n \geq \ell} a_{n} \lambda^{2 n+1} q_{\beta, n-\ell}\right)\right) \\
R_{\alpha,-}(\lambda):= & \exp \left(\epsilon \sqrt{\hbar} \sum_{\beta}\left(\mathfrak{r}_{\ell}\right)_{\alpha \beta} \sum_{n=0}^{\ell-1}(-1)^{n+1} a_{n} \lambda^{2 n+1} \frac{\partial}{\partial q_{\beta, \ell-1-n}}\right) \\
& \times \exp \left(\epsilon \sqrt{\hbar} \sum_{\beta, n \geq 0}\left(\mathfrak{r}_{\ell}\right)_{\alpha \beta} b_{n} \lambda^{-2 n-1} \frac{\partial}{\partial q_{\beta, n+\ell}}\right)
\end{aligned}
$$

Then the $R$-deformed equation (77) turns into

$$
\begin{aligned}
& \operatorname{Res}_{\lambda} \lambda^{2 p}\left(R_{\alpha,+}(\lambda) \Gamma_{\alpha,+}(\lambda) R_{\alpha,-}(\lambda) \Gamma_{\alpha,-}(\lambda) \hat{R} \tau(q)\right. \\
& \left.\otimes R_{\alpha,+}(-\lambda) \Gamma_{\alpha,+}(-\lambda) R_{\alpha,-}(-\lambda) \Gamma_{\alpha,-}(-\lambda) \hat{R} \tau(q)\right) d \lambda=0 .
\end{aligned}
$$

Here $\lambda=\lambda_{\alpha}$ and the equation holds up to $O\left(\epsilon^{2}\right)$.

In a similar way, we define $S_{\alpha,+}(\lambda)$ and $S_{\alpha,-}(\lambda)$ by

$$
\begin{aligned}
S_{\alpha,+}(\lambda):= & \exp \left(\epsilon\left(\mathfrak{s}_{\ell}\right)_{\alpha \alpha} f_{\ell} \lambda^{-2 \ell}\right) \\
& \times \exp \left(\frac{\epsilon}{\sqrt{\hbar}} \sum_{\beta}\left(\mathfrak{s}_{\ell}\right)_{\alpha \beta} \sum_{n=0}^{\ell-1}(-1)^{n} b_{n} \lambda^{-2 n-1} q_{\beta, \ell-1-n}\right) \\
& \times \exp \left(\frac{\epsilon}{\sqrt{\hbar}} \sum_{\beta, n \geq 0}\left(\mathfrak{s}_{\ell}\right)_{\alpha \beta} a_{n} \lambda^{2 n+1} q_{\beta, n+\ell}\right) ; \\
S_{\alpha,-}(\lambda):= & \exp \left(\epsilon \sqrt{\hbar} \sum_{\beta}\left(\mathfrak{s}_{\ell}\right)_{\alpha \beta} \sum_{n \geq \ell}(-1)^{\ell-1} b_{n} \lambda^{-2 n-1} \frac{\partial}{\partial q_{\beta, n-\ell}}\right) .
\end{aligned}
$$

Then the $S$-deformed Hirota equations, obtained by acting with $\hat{S} \otimes \hat{S}$ on (51), turn into

$$
\begin{aligned}
& \operatorname{Res}_{\lambda} \lambda^{2 p}\left(S_{\alpha,+}(\lambda) \Gamma_{\alpha,+}(\lambda) S_{\alpha,-}(\lambda) \Gamma_{\alpha,-}(\lambda) \hat{S} \tau(q)\right. \\
& \left.\otimes S_{\alpha,+}(-\lambda) \Gamma_{\alpha,+}(-\lambda) S_{\alpha,-}(-\lambda) \Gamma_{\alpha,-}(-\lambda) \hat{S} \tau(q)\right) d \lambda=0 .
\end{aligned}
$$

Here again $\lambda=\lambda_{\alpha}$ and the equation holds up to $O\left(\epsilon^{2}\right)$. 
3.5. Global Givental group action on vertex operators. In the previous sections we have computed the first order approximation of the action of the Givental group on vertex operators, and considered the induced infinitesimal deformation of the Hirota equations. In fact, the action of the Givental group can be worked out globally, as follows. First we again write the vertex operator (52) as the quantization

$$
\Gamma_{\alpha}=e^{\hat{\mathfrak{f}}}:=e^{\hat{\mathfrak{f}}_{-}} e^{\hat{\mathfrak{f}}_{+}},
$$

of the function

$$
\mathfrak{f}(z):=\sum_{n=0}^{\infty}\left(-b_{n} \lambda_{\alpha}^{-2 n-1} e_{\alpha} z^{n}-a_{n} \lambda_{\alpha}^{2 n+1} e_{\alpha}(-z)^{-n-1}\right),
$$

and $\mathfrak{f}_{-}$resp. $\mathfrak{f}_{+}$denotes the negative resp. positive powers of $z$. According to $[11, \S 7]$, we have

$$
R e^{\hat{\mathfrak{f}}} R^{-1}=e^{V\left(\mathfrak{f}_{-}^{2}\right) / 2} e^{\widehat{R \mathfrak{f}}},
$$

where the first exponent is an (explicitly computable) constant, which, as we see from (84) only depends on positive powers of $\lambda_{\alpha}$. For $R=$ $\exp \left(\mathfrak{r}_{\ell} z^{\ell}\right), \ell \geq 0$, one computes

$$
e^{\widehat{R f}}=\exp \left(\frac{1}{\sqrt{\hbar}} \sum_{n=0}^{\infty} A_{\alpha, n} q_{\alpha, n}\right) \exp \left(-\sqrt{\hbar} \sum_{n=0}^{\infty} B_{\alpha, n} \frac{\partial}{\partial q_{\alpha, n}}\right),
$$

with

$$
\begin{aligned}
& A_{\alpha, n}=\sum_{\beta} \sum_{k=0}^{\infty} \frac{(-1)^{k \ell}}{k !} a_{k \ell+n} \lambda_{\beta}^{2(k \ell+n)+1}\left(\mathfrak{r}_{\ell}^{k}\right)_{\alpha \beta} \\
& B_{\alpha, n}=\sum_{\beta} \sum_{k=0}^{\infty} \frac{c_{k}^{n}}{k !} \lambda_{\beta}^{2(k \ell-n)-1}\left(\mathfrak{r}_{\ell}^{k}\right)_{\alpha \beta}, \quad c_{k}^{n}= \begin{cases}b_{n-k \ell} & k \ell \leq n \\
(-1)^{k \ell-n} a_{k \ell-n-1} & k \ell>n .\end{cases}
\end{aligned}
$$

We see that the coefficients $A_{\alpha, n}$ and $B_{\alpha, n}$ are power series containing arbitrary positive powers of $\lambda_{\alpha}$. This fact destroys the property of the Hirota equations (22) that, after the change of variables $q_{n}=$ $\xi_{n}+\eta_{n}, q_{n}^{\prime}=\xi_{n}-\eta_{n}$, the power series expansion in $\eta$ has coefficients that are Laurent series in $\lambda_{\alpha}$. Now we have arbitrary formal power series, and the Hirota equations therefore do not lead to a system of finite equations. It is this divergence that forces us to only consider the first order approximations to the Givental action, and look for a "renormalization" of the Hirota equations.

Similarly, the $S$-action can be worked out as (cf. [11, §5])

$$
S e^{\hat{\mathfrak{f}}} S^{-1}=e^{W\left(\mathfrak{f}_{+}^{2}\right) / 2} e^{\widehat{S \mathfrak{f}}},
$$


where $e^{W\left(\mathfrak{f}_{+}^{2}\right) / 2}$ is a similar constant, which now only contains negative powers of $\lambda_{\alpha}$. With $S=\exp \left(\mathfrak{s}_{\ell} z^{-\ell}\right)$, we find

$$
e^{\widehat{S \mathfrak{f}}}=\exp \left(\frac{1}{\sqrt{\hbar}} \sum_{n=0}^{\infty} A_{\alpha, n} q_{\alpha, n}\right) \exp \left(-\sqrt{\hbar} \sum_{n=0}^{\infty} B_{\alpha, n} \frac{\partial}{\partial q_{\alpha, n}}\right),
$$

where the coefficients are now given by

$$
\begin{aligned}
& A_{\alpha, n}=\sum_{\beta} \sum_{k=0}^{\infty} \frac{c_{k}^{n}}{k !} \lambda_{\beta}^{2(n-k \ell)+1}\left(\mathfrak{s}_{\ell}^{k}\right)_{\alpha \beta}, \quad c_{k}^{n}= \begin{cases}(-1)^{n} b_{k \ell-n-1} & k \ell>n \\
(-1)^{k \ell} a_{n-k \ell} & n \leq k \ell .\end{cases} \\
& B_{\alpha, n}=\sum_{\beta} \sum_{k=0}^{\infty} \frac{b_{n+k \ell}}{k !} \lambda_{\beta}^{-2(n+k \ell)-1}\left(\mathfrak{s}_{\ell}^{k}\right)_{\alpha \beta} .
\end{aligned}
$$

This time $A_{\alpha, n}$ and $B_{\alpha, n}$ are bounded above in powers of $\lambda_{\alpha}$, and therefore, the Hirota equations for $S e^{\hat{f}} S^{-1}$ still make sense because in the expansion of $\eta$, we still have power series whose coefficients are Laurent series in $\lambda_{\alpha}$. Therefore, in contrast to the $R$-action, the $S$-action on the Hirota equations is indeed globally defined.

\section{Deformations of the Sato-Wilson equations}

In this section we use the formulas of the previous section to obtain deformations of the Sato-Wilson equations. We follow the procedure described in Section 2 for the KdV hierarchy and multiple copies of the $\mathrm{KdV}$ hierarchy.

4.1. Equations for the deformed wave functions. We first replace in both bilinear equations (80) and (83) the variables $q_{\alpha, 0}$, for $1 \leq \alpha \leq$ $N$, by $q_{\alpha, 0}+x$, so from now on we assume that the tau functions also depend on the variable $x$. Next we divide equation (80) by $\hat{R} \tau(q) \hat{R} \tau\left(q^{\prime}\right)$ and the equation (83) by $\hat{S} \tau(q) \hat{S} \tau\left(q^{\prime}\right)$. This gives the bilinear equation for the deformed wave functions.

Let $G$ be equal to $R$ or $S$, depending on which element in the Givental group we are considering. Introduce the notation

$$
P_{\alpha, G}(\lambda):=\frac{\Gamma_{\alpha,-}(\lambda)(\hat{G} \tau(x, q))}{\hat{G} \tau(x, q)}, \quad \alpha=1, \ldots, N .
$$

We rewrite the bilinear identity (80) or (83) as follows

$$
\operatorname{Res}_{\lambda} \lambda^{2 p} V_{\alpha, G}(x, q, \lambda) e^{\frac{x \lambda}{\sqrt{\hbar}}} V_{\alpha, G}\left(x^{\prime}, q^{\prime},-\lambda\right) e^{\frac{-x^{\prime} \lambda}{\sqrt{\hbar}}} d \lambda=0,
$$

$p=0,1,2, \ldots$, where

$$
V_{\alpha, G}(x, q, \lambda)=G_{\alpha,-}(\lambda)\left(P_{\alpha, G}(\lambda)\right) \frac{G_{\alpha,-}(\lambda)(\hat{G} \tau(x, q))}{\hat{G} \tau(x, q)} G_{\alpha,+}(\lambda) \Gamma_{\alpha,+}(\lambda) e^{\epsilon g_{\alpha, G}(x, \lambda)}
$$


and

$$
g_{\alpha, G}(x, \lambda)= \begin{cases}\frac{(-1)^{\ell-1}}{\sqrt{\hbar}} \sum_{\beta}\left(\mathfrak{r}_{\ell}\right)_{\alpha \beta} a_{\ell} x \lambda^{2 \ell+1} & \text { if } G=R, \\ \frac{(-1)^{\ell-1}}{\sqrt{\hbar}} \sum_{\beta}\left(\mathfrak{s}_{\ell}\right)_{\alpha \beta} b_{\ell-1} x \lambda^{-2 \ell+1} & \text { if } G=S .\end{cases}
$$

4.2. Consequences of the Fundamental Lemma. We now apply the Fundamental Lemma to the bilinear identity (86). We obtain the following system of equations for pseudo-differential operators

$$
\left(V_{\alpha, G}(x, q, \sqrt{\hbar} \partial) \hbar^{p} \partial^{2 p} V_{\alpha, G}\left(x, q^{\prime}, \sqrt{\hbar} \partial\right)^{*}\right)_{-}=0 .
$$

In order to continue we write

$$
V_{\alpha, G}(x, q, \sqrt{\hbar} \partial)=\left(P_{\alpha, G}(\sqrt{\hbar} \partial)+\epsilon Q_{\alpha, G}(x, q, \sqrt{\hbar} \partial)\right) \Gamma_{\alpha,+}(\sqrt{\hbar} \partial)
$$

Clearly as before $\Gamma_{\alpha,+}(\sqrt{\hbar} \partial)^{*}=\Gamma_{\alpha,+}(\sqrt{\hbar} \partial)^{-1}$.

Note also that the deformed tau function $\hat{G} \tau$ does depend on $\epsilon$, since $\hat{G}$ depends on $\epsilon$. However we will make a distintion between equations and possible tau function and $P_{\alpha, G}$ that depends on $\epsilon$.

Substitute (90) into (89); this gives for $p=0,1,2, \ldots$ and $q=q^{\prime}$

$$
\left(\left(P_{\alpha, G}+\epsilon Q_{\alpha, G}\right) \hbar^{p} \partial^{2 p}\left(P_{\alpha, G}^{*}+\epsilon Q_{\alpha, G}^{*}\right)\right)_{-}=0 .
$$

Observe first that, by definition, $\left(P_{\alpha, G}(\sqrt{\hbar} \partial) P_{\alpha, G}(\sqrt{\hbar} \partial)^{*}\right)_{+}=1$. Thus (91) for $p=0$ gives

$$
\epsilon P_{\alpha, G}^{*}=\epsilon P_{\alpha, G}^{-1}
$$

(by this notation we mean that the identity holds at the zeroth order $\epsilon)$. Taking into account also the first order in $\epsilon$ we get

$$
\begin{aligned}
P_{\alpha, G}^{*}+\epsilon Q_{\alpha, G}^{*} & =P_{\alpha, G}^{-1}+\epsilon Q_{\alpha, G}^{*}-\epsilon P_{\alpha, G}^{-1}\left(Q_{\alpha, G} P_{\alpha, G}^{-1}+P_{\alpha, G} Q_{\alpha, G}^{*}\right)_{-} \\
& =P_{\alpha, G}^{-1}\left(1-\epsilon Q_{\alpha, G} P_{\alpha, G}^{-1}+\epsilon\left(Q_{\alpha, G} P_{\alpha, G}^{-1}+P_{\alpha, G} Q_{\alpha, G}^{*}\right)_{+}\right) .
\end{aligned}
$$

4.3. The Lax operator. Define the deformed $\alpha$-th Lax operator as

$$
L_{\alpha, G}:=P_{\alpha, G} \hbar \partial^{2} P_{\alpha, G}^{-1}=L_{\alpha}+\left(L_{\alpha, G}\right)_{-},
$$

where $L_{\alpha}$ denotes its differential part, which is necessarily of the form

$$
L_{\alpha}=\hbar \partial^{2}+2 u_{\alpha}(x, q, \epsilon) .
$$

We stress here that $L_{\alpha}$, and $u_{\alpha}$, do depend on the deformation parameter $\epsilon$, since they are computed from the deformed tau function $\hat{G} \tau$. As we already know, in the undeformed case the negative part of the Lax operator vanishes, hence here it must be at least of order $\epsilon$. In other words $\epsilon L_{\alpha, G}=\epsilon L_{\alpha}$.

Now let's compute how the constraints on the Lax operator look like at the first order in $\epsilon$. Equation (91) for $p=1$ gives

$$
\left(P_{\alpha, G} \hbar \partial^{2} P_{\alpha, G}^{*}+\epsilon Q_{\alpha, G} \hbar \partial^{2} P_{\alpha, G}^{*}+\epsilon P_{\alpha, G} \hbar \partial^{2} Q_{\alpha, G}^{*}\right)_{-}=0
$$


Substituting here (93) we obtain

$$
\left(L_{\alpha, G}-\epsilon\left[L_{\alpha, G}, Q_{\alpha, G} P_{\alpha, G}^{-1}\right]+\epsilon L_{\alpha, G}\left(Q_{\alpha, G} P_{\alpha, G}^{-1}+P_{\alpha, G} Q_{\alpha, G}^{*}\right)_{+}\right)_{-}=0 .
$$

Thus

$$
L_{\alpha, G}=L_{\alpha}+\epsilon\left[L_{\alpha}, Q_{\alpha, G} P_{\alpha, G}^{-1}\right]_{-} .
$$

4.4. The deformed Sato-Wilson equations. In order to obtain the deformed Sato-Wilson equations we differentiate the first component of the Hirota bilinear identity (89) w.r.t. $q_{\beta, n}$ and set $p=0, q=q^{\prime}$,

$$
\begin{gathered}
0=\left(\left(\frac{\partial P_{\alpha, G}}{\partial q_{\beta, n}}+\epsilon \frac{\partial Q_{\alpha, G}}{\partial q_{\beta, n}}+\delta_{\alpha \beta} \frac{a_{n}}{\sqrt{\hbar}}\left(P_{\alpha, G}+\epsilon Q_{\alpha, G}\right)(\sqrt{\hbar} \partial)^{2 n+1}\right)\right. \\
\left.\quad \times\left(P_{\alpha, G}^{*}+\epsilon Q_{\alpha, G}^{*}\right)\right)_{-} \\
=\left(\left(\frac{\partial P_{\alpha, G}}{\partial q_{\beta, n}}+\epsilon \frac{\partial Q_{\alpha, G}}{\partial q_{\beta, n}}+\delta_{\alpha \beta} \frac{a_{n}}{\sqrt{\hbar}}\left(P_{\alpha, G}+\epsilon Q_{\alpha, G}\right)(\sqrt{\hbar} \partial)^{2 n+1}\right) P_{\alpha, G}^{-1}\right. \\
\left.\quad \times\left(1-\epsilon\left(Q_{\alpha, G} P_{\alpha, G}^{-1}-\left(Q_{\alpha, G} P_{\alpha, G}^{-1}+P_{\alpha, G} Q_{\alpha, G}^{*}\right)_{+}\right)\right)\right)_{-} .
\end{gathered}
$$

Thus we see that at the leading order we have the usual Sato-Wilson equations for $N$ copies of the $\mathrm{KdV}$ hierarchy

$$
\frac{\partial P_{\alpha, G}}{\partial q_{\beta, n}} P_{\alpha, G}^{-1}+\delta_{\alpha \beta} \frac{a_{n}}{\sqrt{\hbar}}\left(L_{\alpha, G}^{n+\frac{1}{2}}\right)_{-}=O(\epsilon) .
$$

Using this we can rewrite Equation (99) as

$$
\begin{aligned}
& \frac{\partial P_{\alpha, G}}{\partial q_{\beta, n}} P_{\alpha, G}^{-1}+\delta_{\alpha \beta} \frac{a_{n}}{\sqrt{\hbar}}\left(L_{\alpha, G}^{n+\frac{1}{2}}\right)_{-} \\
& +\epsilon\left(\frac{\partial Q_{\alpha, G} P_{\alpha, G}^{-1}}{\partial q_{\beta, n}}-\delta_{\alpha \beta} \frac{a_{n}}{\sqrt{\hbar}}\left[\left(L_{\alpha}^{n+\frac{1}{2}}\right)_{+}, Q_{\alpha, G} P_{\alpha, G}^{-1}\right]\right)_{-}=0
\end{aligned}
$$

which is a deformation of the Sato-Wilson equations.

In principle one can use this equation (101) to obtain an expression, a Lax type equation, for $\frac{\partial L_{\alpha, G}}{\partial q_{\beta, n}}$.

\subsection{An alternative form of the deformed Sato-Wilson equa-}

tions. Later on it will be convenient to use a different form of the deformed Sato-Wilson equations. We define

$$
\tilde{L}_{\alpha, G}:=L_{\alpha}-\epsilon\left[L_{\alpha}, Q_{\alpha, G} P_{\alpha, G}^{-1}\right]_{+}=L_{\alpha, G}-\epsilon\left[L_{\alpha}, Q_{\alpha, G} P_{\alpha, G}^{-1}\right] .
$$

Since the commutator on the right-hand side is not projected, it is easy to compute the square root of $\widetilde{L}_{\alpha, G}$, and consequently

$$
\left(L_{\alpha, G}^{n+\frac{1}{2}}\right)_{-}=\left(\tilde{L}_{\alpha, G}^{n+\frac{1}{2}}+\epsilon\left[L_{\alpha}^{n+\frac{1}{2}}, Q_{\alpha, G} P_{\alpha, G}^{-1}\right]\right)_{-} .
$$


Substituting in the deformed Sato-Wilson equations (101), we rewrite them as

$$
\begin{aligned}
& \frac{\partial P_{\alpha, G}}{\partial q_{\alpha, p}} P_{\alpha, G}^{-1}+\frac{a_{p}}{\sqrt{\hbar}}\left(\tilde{L}_{\alpha, G}^{p+\frac{1}{2}}\right)_{-} \\
& +\epsilon\left(\frac{\partial Q_{\alpha, G} P_{\alpha, G}^{-1}}{\partial q_{\alpha, p}}+\frac{a_{p}}{\sqrt{\hbar}}\left[\left(L_{\alpha}^{p+\frac{1}{2}}\right)_{-}, Q_{\alpha, G} P_{\alpha, G}^{-1}\right]\right)_{-}=0
\end{aligned}
$$

in the case $\alpha=\beta$. Note that the main advantage of this formula is the different sign of the projector appearing in the commutator.

If $\alpha \neq \beta$, we still have

$$
\frac{\partial P_{\alpha, G}}{\partial q_{\beta, p}} P_{\alpha, G}^{-1}+\epsilon \frac{\partial Q_{\alpha, G} P_{\alpha, G}^{-1}}{\partial q_{\beta, p}}=0 .
$$

4.6. Explicit formulas for $Q_{\alpha, G}$ and $Q_{\alpha, G} P_{\alpha, G}^{-1}$. Now it is straightforward to check that

$$
\begin{aligned}
Q_{\alpha, R} & =\left(\mathfrak{r}_{\ell}\right)_{\alpha \alpha} d_{\ell} P_{\alpha, R}(\sqrt{\hbar} \partial)(\sqrt{\hbar} \partial)^{2 \ell} \\
& +\sqrt{\hbar}\left(\mathfrak{r}_{\ell}\right)_{\alpha \alpha} \sum_{n=0}^{\ell-1}(-1)^{n+1} a_{n} \frac{\partial P_{\alpha, R}}{\partial q_{\alpha, \ell-1-n}}(\sqrt{\hbar} \partial)^{2 n+1} \\
& +\sqrt{\hbar}\left(\mathfrak{r}_{\ell}\right)_{\alpha \alpha} \sum_{n \geq 0} b_{n} \frac{\partial P_{\alpha, R}}{\partial q_{\alpha, n+\ell}}(\sqrt{\hbar} \partial)^{-2 n-1} \\
& +\frac{(-1)^{\ell-1}}{\sqrt{\hbar}} \sum_{\beta}\left(\mathfrak{r}_{\ell}\right)_{\alpha \beta} a_{\ell} x P_{\alpha, R}(\sqrt{\hbar} \partial)(\sqrt{\hbar} \partial)^{2 \ell+1} \\
& +\frac{(-1)^{\ell-1}}{\sqrt{\hbar}} \sum_{\beta}\left(\mathfrak{r}_{\ell}\right)_{\alpha \beta} \sum_{n \geq \ell} a_{n} q_{\beta, n-\ell} P_{\alpha, R}(\sqrt{\hbar} \partial)(\sqrt{\hbar} \partial)^{2 n+1} \\
& +\sqrt{\hbar} \sum_{\beta}\left(\mathfrak{r}_{\ell}\right)_{\alpha \beta} \sum_{n=0}^{\ell-1}(-1)^{n+1} a_{n} \frac{\partial \log \tau}{\partial q_{\beta, \ell-1-n}} P_{\alpha, R}(\sqrt{\hbar} \partial)(\sqrt{\hbar} \partial)^{2 n+1} \\
& +\sqrt{\hbar} \sum_{\beta}\left(\mathfrak{r}_{\ell}\right)_{\alpha \beta} \sum_{n \geq 0} b_{n} \frac{\partial \log \tau}{\partial q_{\beta, \ell+n}} P_{\alpha, R}(\sqrt{\hbar} \partial)(\sqrt{\hbar} \partial)^{-2 n-1} .
\end{aligned}
$$

This formula is obtained by identifying in $(87)$ the $O(\epsilon)$ contributions after substitution of (78),(79), and comparing with the definition (90) of $Q_{\alpha, R}$. One has to carefully take into account the correct ordering of the operators by placing powers of $\lambda$ on the right before substituting $\lambda$ with $\sqrt{\hbar} \partial$.

Note that $Q_{\alpha, G}$ enters the deformed Sato-Wilson equations in terms which are of order at least $\epsilon$. For this reason here we are proving these identities only up to $O(\epsilon)$. 
Similarly we get

$$
\begin{aligned}
Q_{\alpha, S} & =\left(\mathfrak{s}_{\ell}\right)_{\alpha \alpha} f_{\ell} P_{\alpha, S}(\sqrt{\hbar} \partial)(\sqrt{\hbar} \partial)^{-2 \ell} \\
& +\sqrt{\hbar}\left(\mathfrak{s}_{\ell}\right)_{\alpha \alpha} \sum_{n \geq \ell} b_{n} \frac{\partial P_{\alpha, S}}{\partial q_{\alpha, n-\ell}}(\sqrt{\hbar} \partial)^{-2 n-1} \\
& +\frac{1}{\sqrt{\hbar}} \sum_{\beta}\left(\mathfrak{s}_{\ell}\right)_{\alpha \beta}(-1)^{\ell-1} b_{\ell-1} x P_{\alpha, S}(\sqrt{\hbar} \partial)(\sqrt{\hbar} \partial)^{-2 \ell+1} \\
& +\frac{1}{\sqrt{\hbar}} \sum_{\beta}\left(\mathfrak{s}_{\ell}\right)_{\alpha \beta} \sum_{n=0}^{\ell-1}(-1)^{n} b_{n} q_{\beta, \ell-1-n} P_{\alpha, S}(\sqrt{\hbar} \partial)(\sqrt{\hbar} \partial)^{-2 n-1} \\
& +\frac{1}{\sqrt{\hbar}} \sum_{\beta}\left(\mathfrak{s}_{\ell}\right)_{\alpha \beta} \sum_{n \geq 0} a_{n} q_{\beta, \ell+n} P_{\alpha, S}(\sqrt{\hbar} \partial)(\sqrt{\hbar} \partial)^{2 n+1} \\
& +\sqrt{\hbar} \sum_{\beta}\left(\mathfrak{s}_{\ell}\right)_{\alpha \beta}(-1)^{\ell-1} \sum_{n \geq \ell} b_{n} \frac{\partial \log \tau}{\partial q_{\beta, n-\ell}} P_{\alpha, S}(\sqrt{\hbar} \partial)(\sqrt{\hbar} \partial)^{-2 n-1} .
\end{aligned}
$$

Thus, multiplying on the right by $P_{\alpha, G}^{-1}$, we get

$$
\begin{aligned}
Q_{\alpha, R} P_{\alpha, R}^{-1} & =\left(\mathfrak{r}_{\ell}\right)_{\alpha \alpha} d_{\ell} L_{\alpha, R}^{\ell} \\
& -\left(\mathfrak{r}_{\ell}\right)_{\alpha \alpha} \sum_{n=0}^{\ell-1}(-1)^{n+1} a_{n} a_{\ell-1-n}\left(L_{\alpha, R}^{\ell-n-\frac{1}{2}}\right)_{-} L_{\alpha, R}^{n+\frac{1}{2}} \\
& -\left(\mathfrak{r}_{\ell}\right)_{\alpha \alpha} \sum_{n \geq 0} b_{n} a_{n+\ell}\left(L_{\alpha, R}^{n+\ell+\frac{1}{2}}\right)_{-} L_{\alpha, R}^{-n-\frac{1}{2}} \\
& +\frac{(-1)^{\ell-1}}{\sqrt{\hbar}} \sum_{\beta}\left(\mathfrak{r}_{\ell}\right)_{\alpha \beta} a_{\ell} x L_{\alpha, R}^{\ell+\frac{1}{2}} \\
& +\frac{(-1)^{\ell-1}}{\sqrt{\hbar}} \sum_{\beta}\left(\mathfrak{r}_{\ell}\right)_{\alpha \beta} \sum_{n \geq \ell} a_{n} q_{\beta, n-\ell} L_{\alpha, R}^{n+\frac{1}{2}} \\
& +\sqrt{\hbar} \sum_{\beta}\left(\mathfrak{r}_{\ell}\right)_{\alpha \beta} \sum_{n=0}^{\ell-1}(-1)^{n+1} a_{n} \frac{\partial \log \tau}{\partial q_{\beta, \ell-1-n}} L_{\alpha, R}^{n+\frac{1}{2}} \\
& +\sqrt{\hbar} \sum_{\beta}\left(\mathfrak{r}_{\ell}\right)_{\alpha \beta} \sum_{n \geq 0} b_{n} \frac{\partial \log \tau}{\partial q_{\beta, \ell+n}} L_{\alpha, R}^{-n-\frac{1}{2}}
\end{aligned}
$$


and

$$
\begin{aligned}
Q_{\alpha, S} P_{\alpha, S}^{-1} & =\left(\mathfrak{s}_{\ell}\right)_{\alpha \alpha} f_{\ell} L_{\alpha, S}^{-\ell} \\
& -\left(\mathfrak{s}_{\ell}\right)_{\alpha \alpha} \sum_{n \geq \ell} b_{n} a_{n-\ell}\left(L_{\alpha, S}^{n-\ell+\frac{1}{2}}\right)_{-} L_{\alpha, S}^{-n-\frac{1}{2}} \\
& +\frac{1}{\sqrt{\hbar}} \sum_{\beta}\left(\mathfrak{s}_{\ell}\right)_{\alpha \beta}(-1)^{\ell-1} b_{\ell-1} x L_{\alpha, S}^{-\ell+\frac{1}{2}} \\
& +\frac{1}{\sqrt{\hbar}} \sum_{\beta}\left(\mathfrak{s}_{\ell}\right)_{\alpha \beta} \sum_{n=0}^{\ell-1}(-1)^{n} b_{n} q_{\beta, \ell-1-n} L_{\alpha, S}^{-n-\frac{1}{2}} \\
& +\frac{1}{\sqrt{\hbar}} \sum_{\beta}\left(\mathfrak{s}_{\ell}\right)_{\alpha \beta} \sum_{n \geq 0} a_{n} q_{\beta, \ell+n} L_{\alpha, S}^{n+\frac{1}{2}} \\
& +\sqrt{\hbar} \sum_{\beta}\left(\mathfrak{s}_{\ell}\right)_{\alpha \beta}(-1)^{\ell-1} \sum_{n \geq \ell} b_{n} \frac{\partial \log \tau}{\partial q_{\beta, n-\ell}} L_{\alpha, S}^{-n-\frac{1}{2}} .
\end{aligned}
$$

Since we are computing these identities up to terms $O(\epsilon)$, we may substitute everywhere $L_{\alpha, G}$ with $L_{\alpha}$.

4.7. Explicit computation of $S$-deformations. Let us introduce the deformed $\Omega$ functions

$$
\hat{G} \Omega_{\alpha, p ; \beta, q}:=\hbar \frac{\partial^{2} \log \hat{G} \tau}{\partial q_{\alpha, p} \partial q_{\beta, q}} .
$$

As in the undeformed case we get from (85) that

$$
\hat{G} \Omega_{\alpha, 0 ; \beta, p}=-\operatorname{Res}_{\partial} \frac{\partial P_{\alpha, G}}{\partial q_{\beta, q}} P_{\alpha, G}^{-1} .
$$

Now we proceed to substitute in this equation the deformed SatoWilson equations and the explicit formulas for $Q_{\alpha, G} P_{\alpha, G}^{-1}$ obtained above, hence obtaining deformation formulas to be compared to those derived in the Hamiltonian approach in [1].

Let us begin with the $S$-deformations. In the following it is more convenient, from a notational point of view, to consider a general element $\mathfrak{s}=\sum_{\ell \geq 1} \mathfrak{s}_{\ell} z^{-\ell}$ of the lower triangular Lie algebra (rather than fixing $\ell$ ). As before $\hat{S}=e^{\epsilon \hat{\mathfrak{s}}}$.

In the case $\alpha \neq \beta$ we have from Equation (105)

$$
\begin{aligned}
& \frac{\hat{S} \Omega_{\alpha, 0 ; \beta, p}}{\hbar}=\epsilon \operatorname{Res}_{\partial} \frac{\partial Q_{\alpha, S} P_{\alpha, S}^{-1}}{\partial q_{\beta, p}} \\
& =\frac{\epsilon}{\sqrt{\hbar}}\left(\left(\mathfrak{s}_{p+1}\right)_{\alpha \beta} \operatorname{Res}_{\partial} L_{\alpha}^{-\frac{1}{2}}+\sum_{\ell=1}^{p}\left(\mathfrak{s}_{\ell}\right)_{\alpha \beta} a_{p-\ell} \operatorname{Res}_{\partial} L_{\alpha}^{p-\ell+\frac{1}{2}}\right) \\
& =\frac{\epsilon}{\hbar}\left(\left(\mathfrak{s}_{p+1}\right)_{\alpha \beta}+\sum_{\ell=1}^{p}\left(\mathfrak{s}_{\ell}\right)_{\alpha \beta} \Omega_{\alpha, 0 ; \alpha, p-\ell}\right) .
\end{aligned}
$$


Note that this espression has no constant term in $\epsilon$, since in the case of several copies of $\mathrm{KdV}$ the undeformed $\Omega_{\alpha, 0 ; \beta, p}, \alpha \neq \beta$ is equal to zero.

Let $\alpha=\beta$. Then Equation (104) implies that

$$
\text { (113) } \begin{aligned}
\frac{\hat{S} \Omega_{\alpha, 0 ; \alpha, p}}{\hbar}= & \frac{a_{p}}{\sqrt{\hbar}} \operatorname{Res}_{\partial} \tilde{L}_{\alpha, S}^{p+\frac{1}{2}} \\
& +\epsilon \operatorname{Res}_{\partial}\left(\frac{\partial Q_{\alpha, S} P_{\alpha, S}^{-1}}{\partial q_{\alpha, p}}+\frac{a_{p}}{\sqrt{\hbar}}\left[\left(L_{\alpha}^{p+\frac{1}{2}}\right)_{-}, Q_{\alpha, S} P_{\alpha, S}^{-1}\right]\right)
\end{aligned}
$$

This expression requires some further computation.

Lemma 4.1. We have: $a_{p} \sqrt{\hbar} \operatorname{Res}_{\partial} \tilde{L}_{\alpha, S}^{p+\frac{1}{2}}=\Omega_{\alpha, 0 ; \alpha, p}-\epsilon\left(\mathfrak{s}_{1}\right)_{\alpha \mathbb{1}} \Omega_{\alpha, 0 ; \alpha, p-1}$.

Proof. The square root of $\tilde{L}_{\alpha, S}$ is of the form

$$
\tilde{L}_{\alpha, S}^{\frac{1}{2}}=L_{\alpha}^{\frac{1}{2}}+\epsilon Y
$$

where $Y$ is a pseudo-differential operator that solves the equation

$$
Y L_{\alpha}^{\frac{1}{2}}+L_{\alpha}^{\frac{1}{2}} Y=-\left[L, Q_{\alpha, S} P_{\alpha, S}^{-1}\right]_{+} .
$$

It is clear from (109) that

$$
-\left[L_{\alpha}, Q_{\alpha, S} P_{\alpha, S}^{-1}\right]_{+}=-\frac{1}{\sqrt{\hbar}}\left(\left(\mathfrak{s}_{1}\right)_{\alpha \mathbb{1}}\left[L_{\alpha}, x\right] L_{\alpha}^{-\frac{1}{2}}\right)_{+}=-2\left(\mathfrak{s}_{1}\right)_{\alpha \mathbb{1}},
$$

therefore, $Y=-\left(\mathfrak{s}_{1}\right)_{\alpha \Perp} L_{\alpha}^{-\frac{1}{2}}$. Hence we have

$$
\tilde{L}_{\alpha, S}^{p+\frac{1}{2}}=L_{\alpha}^{p+\frac{1}{2}}-\epsilon(2 p+1)\left(s_{1}\right)_{\alpha, \mathbb{1}} L_{\alpha}^{p-\frac{1}{2}} .
$$

Taking the residue of this expression and recalling that

$$
\frac{\Omega_{\alpha, 0 ; \alpha, p}}{\hbar}=\frac{a_{p}}{\sqrt{\hbar}} \operatorname{Res}_{\partial} L_{\alpha}^{p+\frac{1}{2}}
$$

and that $(2 p+1) a_{p}=a_{p-1}$, the statement of the Lemma follows.

The second summand on the right hand side of Equation (113) can be computed directly. The Lax equations imply that at the first order in $\epsilon$ the operator

$$
X \mapsto \frac{\partial X}{\partial q_{\alpha, p}}+\frac{a_{p}}{\sqrt{\hbar}}\left[\left(L_{\alpha}^{p+\frac{1}{2}}\right)_{-}, X\right]
$$

vanishes when applied to any power of $L_{\alpha}$. One can easily check that only a few summands in Equation (109) can contribute to the residue, 
and a direct computation shows that

$$
\begin{aligned}
& \operatorname{Res}_{\partial}\left(\frac{\partial Q_{\alpha, S} P_{\alpha, S}^{-1}}{\partial q_{\alpha, p}}+\frac{a_{p}}{\sqrt{\hbar}}\left[\left(L_{\alpha}^{p+\frac{1}{2}}\right)_{-}, Q_{\alpha, S} P_{\alpha, S}^{-1}\right]\right) \\
& =\frac{\epsilon}{\sqrt{\hbar}}\left(\left(\mathfrak{s}_{p+1}\right)_{\alpha \alpha} \operatorname{Res}_{\partial} L_{\alpha}^{-\frac{1}{2}}+\sum_{\ell=1}^{p}\left(\mathfrak{s}_{\ell}\right)_{\alpha \alpha} a_{p-\ell} \operatorname{Res}_{\partial} L_{\alpha}^{p-\ell+\frac{1}{2}}\right) \\
& =\frac{\epsilon}{\hbar}\left(\left(\mathfrak{s}_{p+1}\right)_{\alpha \alpha}+\sum_{\ell=1}^{p}\left(\mathfrak{s}_{\ell}\right)_{\alpha \alpha} \Omega_{\alpha, 0 ; \alpha, p-\ell}\right) .
\end{aligned}
$$

Summarizing, for $\alpha=\beta$ we have

$$
\hat{S} \Omega_{\alpha, 0 ; \alpha, p}=\Omega_{\alpha, 0 ; \alpha, p}+\epsilon\left(\left(\mathfrak{s}_{p+1}\right)_{\alpha \alpha}+\sum_{\ell=1}^{p}\left(\mathfrak{s}_{\ell}\right)_{\alpha \alpha} \Omega_{\alpha, 0 ; \alpha, p-\ell}-\left(\mathfrak{s}_{1}\right)_{\alpha \mathbb{1}} \Omega_{\alpha, 0 ; \alpha, p-1}\right) .
$$

4.8. Explicit computation of $R$-deformations. Let us now consider the deformation formulas obtained by substituting the Sato-Wilson equations and the explicit formula for $Q_{\alpha, R} P_{\alpha, R}^{-1}$ in equation (111). Here we still consider $\mathfrak{r}=\mathfrak{r}_{\ell} z^{\ell}$ for $\ell$ fixed.

First we consider the case $\alpha \neq \beta$. Equation (105) implies that

$$
\begin{aligned}
& \frac{\hat{R} \Omega_{\alpha, 0 ; \beta, p}}{\hbar}=\epsilon \operatorname{Res}_{\partial} \frac{\partial Q_{\alpha, R} P_{\alpha, R}^{-1}}{\partial q_{\beta, p}} \\
& =\frac{\epsilon\left(\mathfrak{r}_{\ell}\right)_{\alpha \beta}}{\sqrt{\hbar}} \operatorname{Res}_{\partial}\left((-1)^{\ell-1} a_{p+\ell} L_{\alpha}^{p+\ell+\frac{1}{2}}\right. \\
& \left.+\sum_{i=0}^{\ell-1}(-1)^{i+1} a_{i} \Omega_{\beta, \ell-1-i ; \beta, p} L_{\alpha}^{i+\frac{1}{2}}+\Omega_{\beta, \ell ; \beta, p} L_{\alpha}^{-\frac{1}{2}}\right) \\
& =\frac{\epsilon\left(\mathfrak{r}_{\ell}\right)_{\alpha \beta}}{\hbar}\left((-1)^{\ell-1} \Omega_{\alpha, 0 ; \alpha, p+\ell}\right. \\
& \left.+\sum_{i=0}^{\ell-1}(-1)^{i+1} \Omega_{\alpha, 0 ; \alpha, i} \Omega_{\beta, \ell-1-i ; \beta, p}+\Omega_{\beta, \ell ; \beta, p}\right) .
\end{aligned}
$$


In the case $\alpha=\beta$ the residue of the Equation (105) turns into

$$
\begin{aligned}
& \frac{\hat{R} \Omega_{\alpha, 0 ; \alpha, p}}{\hbar}=\frac{a_{p}}{\sqrt{\hbar}} \operatorname{Res}_{\partial}\left(\tilde{L}_{\alpha, R}\right)^{p+\frac{1}{2}} \\
& +\epsilon \operatorname{Res}_{\partial}\left(-\left(\mathfrak{r}_{\ell}\right)_{\alpha \alpha} \sum_{i=0}^{\ell-1}(-1)^{i+1} a_{i} a_{p} \frac{\partial\left(L_{\alpha}^{p+\frac{1}{2}}\right)_{-}}{\partial q_{\alpha, \ell-i-1}} L_{\alpha}^{i+\frac{1}{2}}\right. \\
& +\frac{(-1)^{\ell-1}}{\hbar}\left(\mathfrak{r}_{\ell}\right)_{\alpha \Perp} a_{\ell} a_{p}\left[\left(L_{\alpha}^{p+\frac{1}{2}}\right)_{-}, x\right] L_{\alpha}^{\ell+\frac{1}{2}} \\
& +\frac{1}{\sqrt{\hbar}}\left(\mathfrak{r}_{\ell}\right)_{\alpha \alpha} a_{p+\ell} L_{\alpha}^{p+\ell+\frac{1}{2}} \\
& +\frac{1}{\sqrt{\hbar}}\left(\mathfrak{r}_{\ell}\right)_{\alpha \alpha} \sum_{i=0}^{\ell-1}(-1)^{i+1} a_{i} \Omega_{\alpha, \ell-1-i ; \alpha, p} L_{\alpha}^{i+\frac{1}{2}} \\
& +\sum_{\beta}\left(\mathfrak{r}_{\ell}\right)_{\alpha \beta} \sum_{i=0}^{\ell-1}(-1)^{i+1} a_{i} a_{p}\left[\left(L_{\alpha}^{p+\frac{1}{2}}\right)_{-}, \frac{\partial \log \tau}{\partial q_{\beta, \ell-1-i}}\right] L_{\alpha}^{i+\frac{1}{2}} \\
& \left.+\frac{1}{\sqrt{\hbar}}\left(\mathfrak{r}_{\ell}\right)_{\alpha \alpha} \Omega_{\alpha, \ell ; \alpha, p} L_{\alpha}^{-\frac{1}{2}}\right) .
\end{aligned}
$$

This is a straightforward computation, one has just to use the zerocurvature equations (6).

As in Lemma 4.1, we need to compute the first residue on the righthand side of (123). The square root of $\tilde{L}_{\alpha, R}$ is of the form $\tilde{L}_{\alpha, R}^{\frac{1}{2}}=L_{\alpha}^{\frac{1}{2}}+$ $\epsilon Y$ where $Y$ is a pseudo-differential operator that solves the equation

$$
Y L_{\alpha}^{\frac{1}{2}}+L_{\alpha}^{\frac{1}{2}} Y=-\left[L_{\alpha}, Q_{\alpha, R} P_{\alpha, R}^{-1}\right]_{+} .
$$

Then, we have

$$
a_{p} \sqrt{\hbar} \operatorname{Res}_{\partial} \tilde{L}_{\alpha, R}^{p+\frac{1}{2}}=\Omega_{\alpha, 0 ; \alpha, p}+\epsilon a_{p} \sqrt{\hbar} \operatorname{Res}_{\partial} X
$$

where $X=Y L_{\alpha}^{p}+L_{\alpha}^{\frac{1}{2}} Y L_{\alpha}^{p-\frac{1}{2}}+\cdots+L_{\alpha}^{p} Y$. 
Explicitly, equation (124) becomes

$$
\begin{aligned}
Y L_{\alpha}^{\frac{1}{2}}+L_{\alpha}^{\frac{1}{2}} Y & =\left(\left(\mathfrak{r}_{\ell}\right)_{\alpha \alpha} \sum_{i=0}^{\ell-1}(-1)^{i+1} a_{i} a_{\ell-1-i}\left[L_{\alpha},\left(L_{\alpha}^{\ell-i-\frac{1}{2}}\right)_{-}\right] L_{\alpha}^{i+\frac{1}{2}}\right. \\
& -\frac{(-1)^{\ell-1}}{\sqrt{\hbar}}\left(\mathfrak{r}_{\ell}\right)_{\alpha \mathbb{1}} a_{\ell}\left[L_{\alpha}, x\right] L_{\alpha}^{\ell+\frac{1}{2}} \\
& -\sqrt{\hbar} \sum_{\beta}\left(\mathfrak{r}_{\ell}\right)_{\alpha \beta} \sum_{i=0}^{\ell-1}(-1)^{i+1} a_{i}\left[L_{\alpha}, \frac{\partial \log \tau}{\partial q_{\beta, \ell-1-i}}\right] L_{\alpha}^{i+\frac{1}{2}} \\
& \left.-\sqrt{\hbar} \sum_{\beta}\left(\mathfrak{r}_{\ell}\right)_{\alpha \beta}\left[L_{\alpha}, \frac{\partial \log \tau}{\partial q_{\beta, \ell}}\right] L_{\alpha}^{-\frac{1}{2}}\right)_{+} .
\end{aligned}
$$

Surprisingly enough, this equation, whose solution we could reasonably expect to have only implicitly, has an explicit solution. We give it below, in Lemma 5.2.

\section{Comparison With Deformations in Hamiltonian FORM}

In this section we recall the deformation formulas obtained in [1] in the Hamiltonian approach and compare them with the formulas we have just obtained via deformation of vertex operators.

5.1. The $R$-deformations. We begin with the deformation formula for the $\Omega_{\alpha, p ; \beta, q}$ obtained in [1, Thm. 7].

$$
\begin{aligned}
& \widehat{\mathfrak{r}_{\ell} z^{\ell}}[u] . \Omega_{\alpha, p ; \beta, q}=\left(\mathfrak{r}_{\ell}\right)_{\alpha}^{\mu} \Omega_{\mu, p+\ell ; \beta, q}+\Omega_{\alpha, p ; \mu, q+\ell}\left(\mathfrak{r}_{\ell}\right)_{\beta}^{\mu} \\
& +\sum_{i=0}^{\ell-1}(-1)^{i+1} \Omega_{\alpha, p ; \mu, i}\left(\mathfrak{r}_{\ell}\right)^{\mu \nu} \Omega_{\nu, \ell-1-i ; \beta, q} \\
& \quad-\sum_{\gamma, n} \frac{\partial \Omega_{\alpha, p ; \beta, q}}{\partial u_{\gamma, n}}\left(\left(\mathfrak{r}_{\ell}\right)_{\gamma}^{\mu} \partial_{x}^{n} \Omega_{\mu, \ell ; 1,0}+(n+1) \partial_{x}^{n} \Omega_{\gamma, 0 ; \mu, \ell}\left(\mathfrak{r}_{\ell}\right)_{\mathbb{1}}^{\mu}\right. \\
& \quad+\sum_{i=0}^{\ell-1} \sum_{k=0}^{n-1}\left(\begin{array}{l}
n \\
k
\end{array}\right)(-1)^{i+1} \partial_{x}^{k+1} \Omega_{\gamma, 0 ; \mu, i}\left(\mathfrak{r}_{\ell}\right)^{\mu \nu} \partial_{x}^{n-k-1} \Omega_{\nu, \ell-1-i ; 1,0} \\
& \left.\quad+\sum_{i=0}^{\ell-1}(-1)^{i+1} \partial_{x}^{n}\left(\Omega_{\gamma, 0 ; \mu, i}\left(\mathfrak{r}_{\ell}\right)^{\mu \nu} \Omega_{\nu, \ell-1-i ; 1,0}\right)\right) \\
& +\frac{\hbar}{2} \sum_{\gamma, n} \frac{\partial^{2} \Omega_{\alpha, p ; \beta, q}}{\partial u_{\gamma, n} \partial u_{\zeta, m}} \sum_{i=0}^{\ell-1}(-1)^{i+1} \partial_{x}^{n+1} \Omega_{\gamma, 0 ; \mu, i}\left(\mathfrak{r}_{\ell}\right)^{\mu \nu} \partial_{x}^{m+1} \Omega_{\nu, \ell-1-i ; \zeta, 0}
\end{aligned}
$$


In this formula, a subscript $\mathbb{1}$ means summing over all indices, e.g., $\Omega_{\mu, \ell ; 1,0}=\sum_{\nu} \Omega_{\mu, \ell ; \nu, 0}$, and the following sign convention of [5] is used for raising and lowering indices

$$
\left(\mathfrak{r}_{\ell}\right)^{\beta \alpha}=\left(\mathfrak{r}_{\ell}\right)_{\beta}^{\alpha}=\left(\mathfrak{r}_{\ell}\right)_{\alpha \beta} .
$$

Recall that the symmetry properties of $\mathfrak{r}_{\ell}$ are simply

$$
\left(\mathfrak{r}_{\ell}\right)_{\alpha \beta}=(-1)^{\ell+1}\left(\mathfrak{r}_{\ell}\right)_{\beta \alpha} .
$$

Since we are considering deformations of the tau function of multiple copies the KdV hierarchy, we have that $\Omega_{\alpha, p ; \beta, q}=0$ if $\alpha \neq \beta$.

Moreover it will be sufficient to consider only the case $p=0$, since the deformations of $\Omega_{\alpha, 0 ; \beta, q}$ completely determine the Hamiltonian structure of the deformed hierarchy.

Taking into account these restrictions, the general formula (127), in the case $\alpha \neq \beta$, reduces to

$$
\begin{aligned}
\widehat{\mathfrak{r}_{\ell} z^{\ell}}[u] . \Omega_{\alpha, 0 ; \beta, p}= & (-1)^{\ell-1}\left(\mathfrak{r}_{\ell}\right)_{\alpha \beta}\left(\Omega_{\beta, \ell ; \beta, p}+(-1)^{\ell-1} \Omega_{\alpha, 0 ; \alpha, p+\ell}\right. \\
& \left.+\sum_{i=0}^{\ell-1}(-1)^{i+1} \Omega_{\alpha, 0 ; \alpha, i} \Omega_{\beta, \ell-1-i ; \beta, p}\right)
\end{aligned}
$$

while in the case $\alpha=\beta$ can be rewritten as

$$
\begin{aligned}
& \widehat{\mathfrak{r}_{\ell} z^{\ell}}[u] . \Omega_{\alpha, 0 ; \alpha, p}=\frac{\hbar}{2}\left(\mathfrak{r}_{\ell}\right)_{\alpha \alpha} \sum_{i=0}^{\ell-1}(-1)^{i+1} \frac{\partial^{2} \Omega_{\alpha, 0 ; \alpha, p}}{\partial q_{\alpha, i} \partial q_{\alpha, \ell-1-i}} \\
& +\left(\mathfrak{r}_{\ell}\right)_{\alpha \alpha}\left(\Omega_{\alpha, \ell ; \alpha, p}+\Omega_{\alpha, 0 ; \alpha, p+\ell}+\sum_{i=0}^{\ell-1}(-1)^{i+1} \Omega_{\alpha, 0 ; \alpha, i} \Omega_{\alpha, \ell-1-i ; \alpha, p}\right) \\
& +(-1)^{\ell-1} O \Omega_{\alpha, 0 ; \alpha, p},
\end{aligned}
$$

where $O$ is an operator defined as $O=O_{1}+O_{2}$, where

$$
\begin{aligned}
& O_{1}:=-\sum_{n=0}^{\infty}\left(\sum_{\beta}\left(\mathfrak{r}_{\ell}\right)_{\alpha \beta} \partial_{x}^{n} \Omega_{\beta, \ell ; \beta, 0}+(-1)^{\ell-1}\left(\mathfrak{r}_{\ell}\right)_{\alpha \mathbb{1}}(n+1) \partial_{x}^{n} \Omega_{\alpha, \ell ; \alpha, 0}\right. \\
&\left.+\sum_{\beta}\left(\mathfrak{r}_{\ell}\right)_{\alpha \beta} \sum_{i=0}^{\ell-1}(-1)^{i+1} \sum_{k=0}^{n}\left(\begin{array}{c}
n+1 \\
k
\end{array}\right) \partial_{x}^{k} \Omega_{\alpha, i ; \alpha, 0} \partial_{x}^{n-k} \Omega_{\beta, \ell-1-i ; \beta, 0}\right) \frac{\partial}{\partial u_{\alpha, n}}, \\
& O_{2}:=-\sum_{n=0}^{\infty}\left(\frac{\hbar}{2}\left(\mathfrak{r}_{\ell}\right)_{\alpha \alpha} \sum_{i=0}^{\ell-1}(-1)^{i+1} \partial_{x}^{n+1} \Omega_{\alpha, 0 ; \alpha, i ; \alpha, \ell-1-i}\right) \frac{\partial}{\partial u_{\alpha, n}} .
\end{aligned}
$$

We have denoted $\Omega_{\alpha, p ; \beta, q ; \gamma, r}=\frac{\partial}{\partial q_{\gamma, r}} \Omega_{\alpha, p ; \beta, q}$ and used the identities

$$
u_{\alpha}=\Omega_{\alpha, 0 ; \alpha, 0}, \quad \frac{\partial u_{\alpha, n}}{\partial q_{\alpha, i}}=\partial_{x}^{n+1} \Omega_{\alpha, i ; \alpha, 0}
$$


Theorem 5.1. These deformations coincide with the ones we obtain taking the residue of the Sato-Wilson equation.

Proof. Observe that in the case $\alpha \neq \beta$ the formulas given by Equations (130) and (122) do coincide, up to multiplication by $(-1)^{\ell-1}$, which is exactly the sign we omitted in Equation (67). Therefore we only need to consider the case $\alpha=\beta$, i.e. to show that the complicated deformation formulas given, in the two approaches, by the Equations (123) and (131), are equivalent.

First of all note that the second line in (131) can be written

$$
\begin{aligned}
& \left(\mathfrak{r}_{\ell}\right)_{\alpha \alpha}\left(\Omega_{\alpha, \ell ; \alpha, p}+\Omega_{\alpha, 0 ; \alpha, p+\ell}+\sum_{i=0}^{\ell-1}(-1)^{i+1} \Omega_{\alpha, 0 ; \alpha, i} \Omega_{\alpha, \ell-1-i ; \alpha, p}\right) \\
& =\hbar \operatorname{Res}_{\partial}\left(\frac{1}{\sqrt{\hbar}}\left(\mathfrak{r}_{\ell}\right)_{\alpha \alpha} a_{p+\ell} L_{\alpha}^{p+\ell+\frac{1}{2}}+\frac{1}{\sqrt{\hbar}}\left(\mathfrak{r}_{\ell}\right)_{\alpha \alpha} \Omega_{\alpha, \ell ; \alpha, p} L_{\alpha}^{-\frac{1}{2}}\right. \\
& \left.+\frac{1}{\sqrt{\hbar}}\left(\mathfrak{r}_{\ell}\right)_{\alpha \alpha} \sum_{i=0}^{\ell-1}(-1)^{i+1} a_{i} \Omega_{\alpha, \ell-1-i ; \alpha, p} L_{\alpha}^{i+\frac{1}{2}}\right),
\end{aligned}
$$

reproducing lines 4,5 and 7 in (123).

The operator $O_{1}$ has a nontrivial commutator with $\partial_{x}$ given by

$$
\begin{aligned}
{\left[O_{1}, \partial_{x}\right]=} & -\left((-1)^{\ell-1}\left(\mathfrak{r}_{\ell}\right)_{\alpha \mathbb{1}} \frac{\partial}{\partial q_{\alpha, \ell}}\right. \\
& \left.+\sum_{\beta}\left(\mathfrak{r}_{\ell}\right)_{\alpha \beta} \sum_{i=1}^{\ell-1}(-1)^{i+1} \Omega_{\beta, 0 ; \beta, \ell-1-i} \frac{\partial}{\partial q_{\alpha, i}}\right),
\end{aligned}
$$

where we have used the identities

$$
\left[\frac{\partial}{\partial u_{\alpha, p}}, \partial_{x}\right]=\frac{\partial}{\partial u_{\alpha, n-1}}, \quad \frac{\partial}{\partial q_{\alpha, i}}=\sum_{n \geq 0} \partial_{x}^{n+1} \Omega_{\alpha, 0 ; \alpha, i} \frac{\partial}{\partial u_{\alpha, n}} .
$$

Recalling that

$$
\Omega_{\alpha, 0 ; \alpha, p}=\sqrt{\hbar} a_{p} \operatorname{Res}_{\partial} L_{\alpha}^{p+\frac{1}{2}}
$$

this implies that

$$
\begin{aligned}
& O_{1}\left(\Omega_{\alpha, 0 ; \alpha, p}\right)=\sqrt{\hbar} a_{p} \operatorname{Res}_{\partial}\left[O_{1}, L_{\alpha}^{p+\frac{1}{2}}\right] \\
& =\sqrt{\hbar} a_{p} \operatorname{Res}_{\partial}\left(\left[O_{1}, L_{\alpha}^{\frac{1}{2}}\right] L_{\alpha}^{p}+L_{\alpha}^{\frac{1}{2}}\left[O_{1}, L_{\alpha}^{\frac{1}{2}}\right] L_{\alpha}^{p-\frac{1}{2}}+\cdots+L_{\alpha}^{p}\left[O_{1}, L_{\alpha}^{\frac{1}{2}}\right]\right) .
\end{aligned}
$$

Here $\left[O_{1}, L_{\alpha}^{p+\frac{1}{2}}\right]$ is a pseudo-differential operator in $\partial_{x}$, whose coefficients are differential operators in $\frac{\partial}{\partial q_{\alpha, i}}$. When we take the residue of such an operator, we always mean the coefficient of $\partial_{x}^{-1}$, which does not contain the derivatives $\frac{\partial}{\partial q_{\alpha, i}}$, as one can easily check. 
To proceed, we want to replace $\left[O_{1}, L_{\alpha}^{p+\frac{1}{2}}\right]$ with a usual pseudodifferential operator, whose coefficients are just functions. That is indeed possible by using the operator $Y_{1}$ given by the formula

$$
\begin{aligned}
Y_{1}:= & {\left[O_{1}, L_{\alpha}^{\frac{1}{2}}\right]+\left[L_{\alpha}^{\frac{1}{2}}, x\right](-1)^{\ell-1}\left(\mathfrak{r}_{\ell}\right)_{\alpha \mathbb{1}} \frac{\partial}{\partial q_{\alpha, \ell}} } \\
& +\sum_{\beta}\left(\mathfrak{r}_{\ell}\right)_{\alpha \beta} \sum_{i=1}^{\ell-1}(-1)^{i+1}\left[L_{\alpha}^{\frac{1}{2}}, \hbar \frac{\partial \log \tau}{\partial q_{\beta, \ell-1-i}}\right] \frac{\partial}{\partial q_{\alpha, i}} \\
& -\left[L_{\alpha}^{\frac{1}{2}}, x\right](-1)^{\ell-1}\left(\mathfrak{r}_{\ell}\right)_{\alpha \mathbb{1}} \frac{a_{\ell}}{\sqrt{\hbar}}\left(L_{\alpha}^{\ell+\frac{1}{2}}\right)_{+} \\
& -\sum_{\beta}\left(\mathfrak{r}_{\ell}\right)_{\alpha \beta} \sum_{i=1}^{\ell-1}(-1)^{i+1}\left[L_{\alpha}^{\frac{1}{2}}, \hbar \frac{\partial \log \tau}{\partial q_{\beta, \ell-1-i}}\right] \frac{a_{i}}{\sqrt{\hbar}}\left(L_{\alpha}^{i+\frac{1}{2}}\right)_{+} .
\end{aligned}
$$

First, it follows from Equation (134) that $Y_{1}$ is a pseudo-differential operator. Indeed, the sum of the commutators with $\partial_{x}$ (considered as a factor in $L_{\alpha}^{\frac{1}{2}}$ ) that emerge in the second and the third summands on the right hand side of Equation (137) is equal, with an opposite sign, to the commutator of $O_{1}$ and $\partial_{x}$ given by Equation (134). Second, from the Lax equation (1) it follows that (136) can be rewritten as

$$
\begin{aligned}
& \sqrt{\hbar} a_{p} \operatorname{Res}_{\partial}\left(\left[O_{1}, L_{\alpha}^{\frac{1}{2}}\right] L_{\alpha}^{p}+L_{\alpha}^{\frac{1}{2}}\left[O_{1}, L_{\alpha}^{\frac{1}{2}}\right] L_{\alpha}^{p-\frac{1}{2}}+\cdots+L_{\alpha}^{p}\left[O_{1}, L_{\alpha}^{\frac{1}{2}}\right]\right) \\
& =\sqrt{\hbar} a_{p} \operatorname{Res}_{\partial}\left(\left(Y_{1} L_{\alpha}^{p}+L_{\alpha}^{\frac{1}{2}} Y_{1} L_{\alpha}^{p-\frac{1}{2}}+\cdots+L_{\alpha}^{p} Y_{1}\right)\right. \\
& +\left[L_{\alpha}^{p+\frac{1}{2}}, x\right](-1)^{\ell-1}\left(\mathfrak{r}_{\ell}\right)_{\alpha \mathbb{1}} \frac{a_{\ell}}{\sqrt{\hbar}}\left(L_{\alpha}^{\ell+\frac{1}{2}}\right)_{+} \\
& \left.+\sum_{\beta}\left(\mathfrak{r}_{\ell}\right)_{\alpha \beta} \sum_{i=1}^{\ell-1}(-1)^{i+1}\left[L_{\alpha}^{p+\frac{1}{2}}, \hbar \frac{\partial \log \tau}{\partial q_{\beta, \ell-1-i}}\right] \frac{a_{i}}{\sqrt{\hbar}}\left(L_{\alpha}^{i+\frac{1}{2}}\right)_{+}\right) .
\end{aligned}
$$

Observe that the last two lines give

$$
\begin{aligned}
& \sqrt{\hbar} a_{p} \operatorname{Res}_{\partial}\left(\left[L_{\alpha}^{p+\frac{1}{2}}, x\right](-1)^{\ell-1}\left(\mathfrak{r}_{\ell}\right)_{\alpha \mathbb{1}} \frac{a_{\ell}}{\sqrt{\hbar}}\left(L_{\alpha}^{\ell+\frac{1}{2}}\right)_{+}\right. \\
& \left.+\sum_{\beta}\left(\mathfrak{r}_{\ell}\right)_{\alpha \beta} \sum_{i=1}^{\ell-1}(-1)^{i+1}\left[L_{\alpha}^{p+\frac{1}{2}}, \hbar \frac{\partial \log \tau}{\partial q_{\beta, \ell-1-i}}\right] \frac{a_{i}}{\sqrt{\hbar}}\left(L_{\alpha}^{i+\frac{1}{2}}\right)_{+}\right) \\
& =\sqrt{\hbar} a_{p} \operatorname{Res}_{\partial}\left(\left[\left(L_{\alpha}^{p+\frac{1}{2}}\right)_{-}, x\right](-1)^{\ell-1}\left(\mathfrak{r}_{\ell}\right)_{\alpha \mathbb{1}} \frac{a_{\ell}}{\sqrt{\hbar}}\left(L_{\alpha}^{\ell+\frac{1}{2}}\right)\right. \\
& \left.+\sum_{\beta}\left(\mathfrak{r}_{\ell}\right)_{\alpha \beta} \sum_{i=1}^{\ell-1}(-1)^{i+1}\left[\left(L_{\alpha}^{p+\frac{1}{2}}\right)_{-}, \hbar \frac{\partial \log \tau}{\partial q_{\beta, \ell-1-i}}\right] \frac{a_{i}}{\sqrt{\hbar}}\left(L_{\alpha}^{i+\frac{1}{2}}\right)\right),
\end{aligned}
$$

so in this way we identify other two summands in Equation (123). 
The remaining terms of Equation (131) can be rewritten in the following way:

$$
\begin{aligned}
& \frac{\hbar}{2}\left(\mathfrak{r}_{\ell}\right)_{\alpha \alpha} \sum_{i=0}^{\ell-1}(-1)^{i+1} \frac{\partial^{2} \Omega_{\alpha, 0 ; \alpha, p}}{\partial q_{\alpha, i} \partial q_{\alpha, \ell-1-i}}+O_{2} \Omega_{\alpha, 0 ; \alpha, p} \\
& =\sqrt{\hbar} a_{p} \operatorname{Res}_{\partial}\left(Y_{2} L_{\alpha}^{p}+L_{\alpha}^{\frac{1}{2}} Y_{2} L_{\alpha}^{p-\frac{1}{2}}+\cdots+L_{\alpha}^{p} Y_{2}\right. \\
& \left.+\hbar\left(\mathfrak{r}_{\ell}\right)_{\alpha \alpha} \sum_{i=0}^{\ell-1}(-1)^{i+1} \sum_{a+b+c=2 p-1}\left(L_{\alpha}^{\frac{1}{2}}\right)^{a} \frac{\partial L_{\alpha}^{\frac{1}{2}}}{\partial q_{\alpha, i}}\left(L_{\alpha}^{\frac{1}{2}}\right)^{b} \frac{\partial L_{\alpha}^{\frac{1}{2}}}{\partial q_{\alpha, i}}\left(L_{\alpha}^{\frac{1}{2}}\right)^{c}\right)
\end{aligned}
$$

where

$$
\begin{aligned}
& Y_{2}:=\frac{\hbar}{2}\left(\mathfrak{r}_{\ell}\right)_{\alpha \alpha} \sum_{i=0}^{\ell-1}(-1)^{i+1} \frac{\partial^{2}\left(L_{\alpha}^{\frac{1}{2}}\right)}{\partial q_{\alpha, i} \partial q_{\alpha, \ell-1-i}} \\
& {\left[-\sum_{n=0}^{\infty}\left(\frac{\hbar}{2}\left(\mathfrak{r}_{\ell}\right)_{\alpha \alpha} \sum_{i=0}^{\ell-1}(-1)^{i+1} \partial_{x}^{n+1} \Omega_{\alpha, 0 ; \alpha, i ; \alpha, \ell-1-i}\right) \frac{\partial}{\partial u_{\alpha, n}}, L_{\alpha}^{\frac{1}{2}}\right]}
\end{aligned}
$$

Meanwhile, using the Lax equation (1), we see that the last term in $(140)$ is

$$
\begin{aligned}
& \sqrt{\hbar} a_{p} \operatorname{Res}_{\partial} \hbar\left(\mathfrak{r}_{\ell}\right)_{\alpha \alpha} \sum_{i=0}^{\ell-1}(-1)^{i+1} \sum_{\substack{a+b+c \\
=2 p-1}}\left(L_{\alpha}^{\frac{1}{2}}\right)^{a} \frac{\partial L_{\alpha}^{\frac{1}{2}}}{\partial q_{\alpha, i}}\left(L_{\alpha}^{\frac{1}{2}}\right)^{b} \frac{\partial L_{\alpha}^{\frac{1}{2}}}{\partial q_{\alpha, i}}\left(L_{\alpha}^{\frac{1}{2}}\right)^{c} \\
& =\sqrt{\hbar} a_{p} \operatorname{Res}_{\partial}\left(Y_{3} L_{\alpha}^{p}+L_{\alpha}^{\frac{1}{2}} Y_{3} L_{\alpha}^{p-\frac{1}{2}}+\cdots+L_{\alpha}^{p} Y_{3}\right) \\
& -\sqrt{\hbar} a_{p} \operatorname{Res}_{\partial} \hbar\left(\mathfrak{r}_{\ell}\right)_{\alpha \alpha} \sum_{i=0}^{\ell-1}(-1)^{i+1} \frac{\partial\left(L_{\alpha}^{p+\frac{1}{2}}\right)}{\partial q_{\alpha, i}} \frac{a_{\ell-1-i}}{\sqrt{\hbar}}\left(L_{\alpha}^{\ell-i-\frac{1}{2}}\right)_{+} .
\end{aligned}
$$

The last line is equal to

$$
-\hbar \operatorname{Res}_{\partial}\left(\mathfrak{r}_{\ell}\right)_{\alpha \alpha} \sum_{i=0}^{\ell-1}(-1)^{i+1} a_{i} a_{p} \frac{\partial\left(L_{\alpha}^{p+\frac{1}{2}}\right)}{\partial q_{\alpha, \ell-i-1}} L_{\alpha}^{i+\frac{1}{2}},
$$

which corresponds to the last summand in Equation (123) that we haven't yet reproduced, and $Y_{3}$ is given by the formula

$$
Y_{3}:=\hbar\left(\mathfrak{r}_{\ell}\right)_{\alpha \alpha} \sum_{i=0}^{\ell-1}(-1)^{i+1} \frac{\partial\left(L_{\alpha}^{\frac{1}{2}}\right)}{\partial q_{\alpha, i}} \frac{a_{\ell-1-i}}{\sqrt{\hbar}}\left(L_{\alpha}^{\ell-i-\frac{1}{2}}\right)_{+} .
$$

If we show that $Y:=Y_{1}+Y_{2}+Y_{3}$ solves (124) then the contribution from the terms in (138), (140), (142) that we have not matched yet, 
i.e.

$$
\sqrt{\hbar} a_{p} \operatorname{Res}_{\partial}\left(Y L_{\alpha}^{p}+L_{\alpha}^{\frac{1}{2}} Y L_{\alpha}^{p-\frac{1}{2}}+\cdots+L_{\alpha}^{p} Y\right)
$$

gives exactly the deformation term of (125).

So, in order to complete the proof of the theorem, it is sufficient to show that $Y:=Y_{1}+Y_{2}+Y_{3}$ is a solution of the Equation (124). This we do below, in Lemma 5.2.

Lemma 5.2. The pseudo-differential operator $Y:=Y_{1}+Y_{2}+Y_{3}$ solves Equation (124).

Proof. First, observe that

$$
\begin{aligned}
& L_{\alpha}^{\frac{1}{2}} Y_{1}+Y_{1} L_{\alpha}^{\frac{1}{2}}=\left[O_{1}, L_{\alpha}\right] \\
& +\left[L_{\alpha}, x\right](-1)^{\ell-1}\left(\mathfrak{r}_{\ell}\right)_{\alpha \mathbb{1}} \frac{\partial}{\partial q_{\alpha, \ell}}+\left[L_{\alpha}^{\frac{1}{2}}, x\right](-1)^{\ell-1}\left(\mathfrak{r}_{\ell}\right)_{\alpha \mathbb{1}} \frac{\partial L_{\alpha}^{\frac{1}{2}}}{\partial q_{\alpha, \ell}} \\
& +\sum_{\beta}\left(\mathfrak{r}_{\ell}\right)_{\alpha \beta} \sum_{i=1}^{\ell-1}(-1)^{i+1}\left[L_{\alpha}, \hbar \frac{\partial \log \tau}{\partial q_{\beta, \ell-1-i}}\right] \frac{\partial}{\partial q_{\alpha, i}} \\
& +\sum_{\beta}\left(\mathfrak{r}_{\ell}\right)_{\alpha \beta} \sum_{i=1}^{\ell-1}(-1)^{i+1}\left[L_{\alpha}^{\frac{1}{2}}, \hbar \frac{\partial \log \tau}{\partial q_{\beta, \ell-1-i}}\right] \frac{\partial L_{\alpha}^{\frac{1}{2}}}{\partial q_{\alpha, i}} \\
& -\left[L_{\alpha}, x\right](-1)^{\ell-1}\left(\mathfrak{r}_{\ell}\right)_{\alpha \mathbb{1}} \frac{a_{\ell}}{\sqrt{\hbar}}\left(L_{\alpha}^{\ell+\frac{1}{2}}\right)_{+} \\
& -\left[L_{\alpha}^{\frac{1}{2}}, x\right](-1)^{\ell-1}\left(\mathfrak{r}_{\ell}\right)_{\alpha \mathbb{1}} \frac{a_{\ell}}{\sqrt{\hbar}}\left[\left(L_{\alpha}^{\ell+\frac{1}{2}}\right)_{+}, L_{\alpha}^{\frac{1}{2}}\right] \\
& -\sum_{\beta}\left(\mathfrak{r}_{\ell}\right)_{\alpha \beta} \sum_{i=1}^{\ell-1}(-1)^{i+1}\left[L_{\alpha}, \hbar \frac{\partial \log \tau}{\partial q_{\beta, \ell-1-i}}\right] \frac{a_{i}}{\sqrt{\hbar}}\left(L_{\alpha}^{i+\frac{1}{2}}\right)_{+} \\
& -\sum_{\beta}\left(\mathfrak{r}_{\ell}\right)_{\alpha \beta} \sum_{i=1}^{\ell-1}(-1)^{i+1}\left[L_{\alpha}^{\frac{1}{2}}, \hbar \frac{\partial \log \tau}{\partial q_{\beta, \ell-1-i}}\right] \frac{a_{i}}{\sqrt{\hbar}}\left[\left(L_{\alpha}^{i+\frac{1}{2}}\right)_{+}, L_{\alpha}^{\frac{1}{2}}\right] .
\end{aligned}
$$


Using the Lax equation (59) and the explicit formulas for $O_{1}$ and $L_{\alpha}$ (Equations (132) and (57), respectively), we can rewrite this as

$$
\begin{aligned}
& L_{\alpha}^{\frac{1}{2}} Y_{1}+Y_{1} L_{\alpha}^{\frac{1}{2}}=\left[L_{\alpha}, x\right](-1)^{\ell-1}\left(\mathfrak{r}_{\ell}\right)_{\alpha \mathbb{1}} \frac{\partial}{\partial q_{\alpha, \ell}} \\
& +\sum_{\beta}\left(\mathfrak{r}_{\ell}\right)_{\alpha \beta} \sum_{i=1}^{\ell-1}(-1)^{i+1}\left[L_{\alpha}, \hbar \frac{\partial \log \tau}{\partial q_{\beta, \ell-1-i}}\right] \frac{\partial}{\partial q_{\alpha, i}} \\
& -\left[L_{\alpha}, x\right](-1)^{\ell-1}\left(\mathfrak{r}_{\ell}\right)_{\alpha \mathbb{1}} \frac{a_{\ell}}{\sqrt{\hbar}}\left(L_{\alpha}^{\ell+\frac{1}{2}}\right)_{+} \\
& -\sum_{\beta}\left(\mathfrak{r}_{\ell}\right)_{\alpha \beta} \sum_{i=1}^{\ell-1}(-1)^{i+1}\left[L_{\alpha}, \hbar \frac{\partial \log \tau}{\partial q_{\beta, \ell-1-i}}\right] \frac{a_{i}}{\sqrt{\hbar}}\left(L_{\alpha}^{i+\frac{1}{2}}\right)_{+} \\
& -2 \cdot\left(\sum_{\beta}\left(\mathfrak{r}_{\ell}\right)_{\alpha \beta} \Omega_{\beta, \ell ; \beta, 0}+(-1)^{\ell-1}\left(\mathfrak{r}_{\ell}\right)_{\alpha \mathbb{1}} \Omega_{\alpha, \ell ; \alpha, 0}\right. \\
& \left.+\sum_{\beta}\left(\mathfrak{r}_{\ell}\right)_{\alpha \beta} \sum_{i=0}^{\ell-1}(-1)^{i+1} \Omega_{\alpha, i ; \alpha, 0} \Omega_{\beta, \ell-1-i ; \beta, 0}\right) \\
& -2 \hbar\left((-1)^{\ell-1}\left(\mathfrak{r}_{\ell}\right)_{\alpha \mathbb{1}} \frac{\partial}{\partial q_{\alpha, \ell}}\right. \\
& \left.+\sum_{\beta}\left(\mathfrak{r}_{\ell}\right)_{\alpha \beta} \sum_{i=1}^{\ell-1}(-1)^{i+1} \Omega_{\beta, 0 ; \beta, \ell-1-i} \frac{\partial}{\partial q_{\alpha, i}}\right) \partial_{x} \\
& -\hbar \sum_{\beta}\left(\mathfrak{r}_{\ell}\right)_{\alpha \beta} \sum_{i=1}^{\ell-1}(-1)^{i+1} \partial_{x} \Omega_{\beta, 0 ; \beta, \ell-1-i} \frac{\partial}{\partial q_{\alpha, i}} \\
& =-\left[L_{\alpha}, x\right](-1)^{\ell-1}\left(\mathfrak{r}_{\ell}\right)_{\alpha \mathbb{1}} \frac{a_{\ell}}{\sqrt{\hbar}}\left(L_{\alpha}^{\ell+\frac{1}{2}}\right)_{+} \\
& -\sum_{\beta}\left(\mathfrak{r}_{\ell}\right)_{\alpha \beta} \sum_{i=1}^{\ell-1}(-1)^{i+1}\left[L_{\alpha}, \hbar \frac{\partial \log \tau}{\partial q_{\beta, \ell-1-i}}\right] \frac{a_{i}}{\sqrt{\hbar}}\left(L_{\alpha}^{i+\frac{1}{2}}\right)_{+} \\
& -2 \cdot\left(\sum_{\beta}\left(\mathfrak{r}_{\ell}\right)_{\alpha \beta} \Omega_{\beta, \ell ; \beta, 0}+(-1)^{\ell-1}\left(\mathfrak{r}_{\ell}\right)_{\alpha \mathbb{1}} \Omega_{\alpha, \ell ; \alpha, 0}\right. \\
& \left.+\sum_{\beta}\left(\mathfrak{r}_{\ell}\right)_{\alpha \beta} \sum_{i=0}^{\ell-1}(-1)^{i+1} \Omega_{\alpha, i ; \alpha, 0} \Omega_{\beta, \ell-1-i ; \beta, 0}\right) \text {. }
\end{aligned}
$$


In order to match this expression to the right hand side of the Equation (124), we note that

$$
\begin{aligned}
& \left(\frac{a_{\ell}}{\sqrt{\hbar}}\left[L_{\alpha}, x\right] L_{\alpha}^{\ell+\frac{1}{2}}\right)_{+}=2 \Omega_{\alpha, 0 ; \alpha, \ell}+\frac{a_{\ell}}{\sqrt{\hbar}}\left[L_{\alpha}, x\right]\left(L_{\alpha}^{\ell+\frac{1}{2}}\right)_{+}, \\
& \left(\frac{a_{i}}{\sqrt{\hbar}}\left[L_{\alpha}, \frac{\hbar \partial \log \tau}{\partial q_{\beta, j}}\right] L_{\alpha}^{i+\frac{1}{2}}\right)_{+}=2 \Omega_{\alpha, i ; \alpha, 0} \Omega_{\beta, j ; \beta, 0} \\
& +\left[L_{\alpha}, \frac{\hbar \partial \log \tau}{\partial q_{\beta, j}}\right] \frac{a_{i}}{\sqrt{\hbar}}\left(L_{\alpha}^{i+\frac{1}{2}}\right)_{+}, \\
& \left(\sqrt{\hbar}\left[L_{\alpha}, \frac{\partial \log \tau}{\partial q_{\beta, \ell}}\right] L_{\alpha}^{-\frac{1}{2}}\right)_{+}=2 \Omega_{\beta, \ell ; \beta, 0} \cdot
\end{aligned}
$$

Then we observe that

$$
\begin{aligned}
& L_{\alpha}^{\frac{1}{2}}\left(Y_{2}+Y_{3}\right)+\left(Y_{2}+Y_{3}\right) L_{\alpha}^{\frac{1}{2}}= \\
& \frac{\hbar}{2}\left(\mathfrak{r}_{\ell}\right)_{\alpha \alpha} \sum_{i=0}^{\ell-1}(-1)^{i+1}\left(\frac{\partial^{2}\left(L_{\alpha}^{\frac{1}{2}}\right)}{\partial q_{\alpha, i} \partial q_{\alpha, \ell-1-i}} L_{\alpha}^{\frac{1}{2}}+L_{\alpha}^{\frac{1}{2}} \frac{\partial^{2}\left(L_{\alpha}^{\frac{1}{2}}\right)}{\partial q_{\alpha, i} \partial q_{\alpha, \ell-1-i}}\right) \\
& -2\left(\frac{\hbar}{2}\left(\mathfrak{r}_{\ell}\right)_{\alpha \alpha} \sum_{i=0}^{\ell-1}(-1)^{i+1} \partial_{x} \Omega_{\alpha, 0 ; \alpha, i ; \alpha, \ell-1-i}\right) \\
& +\hbar\left(\mathfrak{r}_{\ell}\right)_{\alpha \alpha} \sum_{i=0}^{\ell-1}(-1)^{i+1} \frac{\partial\left(L_{\alpha}^{\frac{1}{2}}\right)}{\partial q_{\alpha, i}} \frac{a_{\ell-1-i}}{\sqrt{\hbar}}\left[\left(L_{\alpha}^{\ell-i-\frac{1}{2}}\right)_{+}, L_{\alpha}^{\frac{1}{2}}\right] \\
& +\hbar\left(\mathfrak{r}_{\ell}\right)_{\alpha \alpha} \sum_{i=0}^{\ell-1}(-1)^{i+1} \frac{\partial L_{\alpha}}{\partial q_{\alpha, i}} \frac{a_{\ell-1-i}}{\sqrt{\hbar}}\left(L_{\alpha}^{\ell-i-\frac{1}{2}}\right)_{+} .
\end{aligned}
$$

The sum of the first three summands on the right hand side of this equation is equal to zero. The last summand is a differential operator, that is, has no negative part, and the Lax equations imply that

$$
\begin{aligned}
& \hbar\left(\mathfrak{r}_{\ell}\right)_{\alpha \alpha} \sum_{i=0}^{\ell-1}(-1)^{i+1} \frac{\partial L_{\alpha}}{\partial q_{\alpha, i}} \frac{a_{\ell-1-i}}{\sqrt{\hbar}}\left(L_{\alpha}^{\ell-i-\frac{1}{2}}\right)_{+} \\
& =\left(\left(\mathfrak{r}_{\ell}\right)_{\alpha \alpha} \sum_{i=0}^{\ell-1}(-1)^{i+1} a_{i} a_{\ell-1-i}\left[L_{\alpha},\left(L_{\alpha}^{\ell-i-\frac{1}{2}}\right)_{-}\right] L_{\alpha}^{i+\frac{1}{2}}\right)_{+},
\end{aligned}
$$

which is the only remaining summand on the right hand side of Equation (124).

Thus we see that $Y_{1}+Y_{2}+Y_{3}$ solves Equation (124).

5.2. The $S$-deformations. We recall the formula for the $S$-deformation of $\Omega_{\alpha, p ; \beta, q}$ obtained in [1, Thm. 9]: 


$$
\begin{aligned}
& \sum_{\ell=1}^{\infty} \widehat{\mathfrak{s}_{\ell} z^{-\ell}}[u] . \Omega_{\alpha, p ; \beta, q}=\sum_{1 \leq \ell \leq p}\left(\mathfrak{s}_{\ell}\right)_{\alpha}^{\mu} \Omega_{\mu, p-\ell ; \beta, q}+\sum_{1 \leq \ell \leq q} \Omega_{\alpha, p ; \mu, q-\ell}\left(\mathfrak{s}_{\ell}\right)_{\beta}^{\mu} \\
& +(-1)^{p}\left(\mathfrak{s}_{p+q+1}\right)_{\alpha \beta}-\sum_{\gamma} \frac{\partial \Omega_{\alpha, p ; \beta, q}}{\partial u_{\gamma, 0}}\left(\mathfrak{s}_{1}\right)_{\gamma, \mathbb{1}} .
\end{aligned}
$$

As before indices are raised and lowered according to the rule (128).

In the special case of several copies of $\mathrm{KdV}$, and for $p=0$, this formula can be simplified. Indeed, if $\alpha \neq \beta$, then

$$
\sum_{\ell=1}^{\infty} \widehat{\mathfrak{s}_{\ell} z^{-\ell}}[u] . \Omega_{\alpha, 0 ; \beta, p}=\sum_{\ell=1}^{p}\left(\mathfrak{s}_{\ell}\right)_{\alpha \beta} \Omega_{\alpha, 0 ; \alpha, p-\ell}+\left(\mathfrak{s}_{p+1}\right)_{\alpha \beta} .
$$

In the case $\alpha=\beta$, we have

$$
\begin{aligned}
\sum_{\ell=1}^{\infty} \widehat{\mathfrak{s}_{\ell} z^{-\ell}}[u] . \Omega_{\alpha, 0 ; \alpha, p}= & \sum_{\ell=1}^{p}\left(\mathfrak{s}_{\ell}\right)_{\alpha \alpha} \Omega_{\alpha, 0 ; \alpha, p-\ell}+\left(\mathfrak{s}_{p+1}\right)_{\alpha \alpha} \\
& -\left(\mathfrak{s}_{1}\right)_{\alpha, \mathbb{1}} \Omega_{\alpha, 0 ; \alpha, p-1} .
\end{aligned}
$$

Theorem 5.3. These deformations coincide with the ones we obtain taking the residue of the Sato-Wilson equation.

Proof. Indeed, the right hand side of Equation (152) is equal to the right hand side of Equation (112). From Lemma 4.1 and Equation (120) it follows that the $\epsilon$-term on the right hand side of Equation (113) multiplied by $\hbar$ is equal to the right hand side of Equation (153).

\section{REFERENCES}

[1] A. Buryak, H. Posthuma, S. Shadrin, A polynomial bracket for the DubrovinZhang hierarchies, arXiv: 1009.5351, $31 \mathrm{pp.}$

[2] A. Buryak, H. Posthuma, S. Shadrin, On deformations of quasi-Miura transformations and the Dubrovin-Zhang bracket, arXiv:1104.2722, 21 pp.

[3] L. A. Dickey, Soliton equations and Hamiltonian systems. Second edition. Advanced Series in Mathematical Physics, 26. World Scientific Publishing Co., Inc., River Edge, NJ, 2003.

[4] B. A. Dubrovin, Y. Zhang, Normal forms of hierarchies of integrable PDEs, Frobenius manifolds and Gromov-Witten invariants, a new 2005 version of arXiv:math/0108160v1, 295 pp.

[5] Faber, Carel; Shadrin, Sergey; Zvonkine, Dimitri. Tautological relations and the $r$-spin Witten conjecture. Ann. Sci. c. Norm. Supr. (4) 43 (2010), no. 4, $621-658$.

[6] Huijun Fan, Tyler J. Jarvis, Yongbin Ruan, The Witten equation and its virtual fundamental cycle arXiv:0712.4025, $108 \mathrm{pp}$.

[7] V. G. Kac, J. W. van de Leur, The n-component KP hierarchy and representation theory. Integrability, topological solitons and beyond. J. Math. Phys. 44 (2003), no. 8, 3245-3293.

[8] Givental, Alexander B. Gromov-Witten invariants and quantization of quadratic Hamiltonians. Dedicated to the memory of I. G. Petrovskii on the occasion of his 100th anniversary. Mosc. Math. J. 1 (2001), no. 4, 551-568, 645. 
[9] Givental, Alexander B. Semisimple Frobenius structures at higher genus. Internat. Math. Res. Notices 2001, no. 23, 1265-1286.

[10] Givental, Alexander B. Symplectic geometry of Frobenius structures. In; Frobenius manifolds, 91-112, Aspects Math., E36, Vieweg, Wiesbaden, 2004.

[11] Givental, Alexander B. $A_{n-1}$ singularities and $n \mathrm{KdV}$ hierarchies. Mosc. Math. J. 3 (2003), no. 2, 475505

[12] Givental, Alexander B.; Milanov, Todor E. Simple singularities and integrable hierarchies. In; The breadth of symplectic and Poisson geometry, 173-201, Progr. Math., 232, Birkhuser Boston, Boston, MA, 2005.

[13] Lee, Y.-P. Invariance of tautological equations. I. Conjectures and applications. J. Eur. Math. Soc. (JEMS) 10 (2008), no. 2, 399-413.

[14] Lee, Y.-P. Invariance of tautological equations. II. Gromov-Witten theory. With an appendix by Y. Iwao and the author. J. Amer. Math. Soc. 22 (2009), no. $2,331-352$.

[15] Milanov, Todor E. Hirota quadratic equations for the extended Toda hierarchy. Duke Math. J. 138 (2007), no. 1, 161178.

[16] Milanov, Todor E. The equivariant Gromov-Witten theory of $\mathbb{C P}^{1}$ and integrable hierarchies. Int. Math. Res. Not. 2008

[17] Milanov, Todor E.; Tseng, Hsian-Hua. The spaces of Laurent polynomials, Gromov-Witten theory of $\mathbb{P}^{1}$-orbifolds, and integrable hierarchies. J. Reine Angew. Math. 622 (2008), 189235.

[18] Shadrin, Sergey. BCOV theory via Givental group action on cohomological fields theories. Mosc. Math. J. 9 (2009), no. 2, 411-429.

G. Carlet: Dipartimento di Matematica e Applicazioni, Università di Milano-Bicocca, Via R. Cozzi, 53, 20125 Milano, Italia

E-mail address: guido.carlet@unimib.it

J. van de Leur: Mathematisch Insituut, Universiteit Utrecht, PostBus 80010, 3508 TA Utrecht, Nederland

E-mail address: j.w.vandeleur@uu.nl

H. Posthuma: Korteweg-De Vries Instituut voor Wiskunde, UniverSiteit van Amsterdam, Postbus 94248, 1090 GE Amsterdam, Nederland E-mail address: h.b.posthuma@uva.nl

S. Shadrin: Korteweg-de Vries Institudt voor Wiskunde, Universiteit van Amsterdam, Postbus 94248, 1090 GE Amsterdam, Nederland E-mail address: s.shadrin@uva.nl 